\title{
PERTURBATION THEORY FOR THE LAPLACIAN ON AUTOMORPHIC FUNCTIONS
}

\author{
R. PHILLIPS AND P. SARNAK
}

\section{INTRODUCTION}

Let $\Gamma \subset \operatorname{PSL}(2, \mathbb{R})$ be a discrete subgroup, whose quotient $\Gamma \backslash H$ ( $H$ being the upper halfplane) is of finite volume but not compact. In studying perturbation theory for the Laplacian as $\Gamma$ moves in Teichmüller space $T(\Gamma)$, it is best not to limit oneself to the eigenvalues of the Laplacian since they are very unstable [PS1]. Instead we shall consider a larger set of values associated with $\Gamma$, which we call the singular set $\sigma(\Gamma)$; this set is well behaved under deformation. Our approach to this problem makes use of the wave equation [LP1], which provides a convenient framework for the study of the singular set under perturbations.

To motivate the definition of the singular set consider the following form of the trace formula (our notation is the same as Selberg's [Se]). It can be easily derived form the usual formula by means of Cauchy's residue theorem and some straightforward manipulations (cf. [LP1, Theorem 9.10]).

Let $g \in C_{0}^{(\infty)}\left(\mathbb{R}_{+}\right)$(it is essential that $g$ be supported in $(0, \infty)$ rather than the usual choice of $\mathbb{R}$ ) and let $h=\hat{g}$. Assume also that $\Gamma$ contains no elliptic elements. Then

$$
\begin{aligned}
\sum_{j} h\left(r_{j}\right) & +\sum_{j} h\left(i\left(\frac{1}{2}-\rho_{j}\right)\right)+\frac{n+\operatorname{tr}[\Phi(1 / 2)]}{2} h(0) \\
= & |F| \int_{-\infty}^{\infty} h(r) r \tanh (\pi r) d r-\frac{n}{2 \pi} \int_{-\infty}^{\infty}(h(r)+h(-r)) \frac{\Gamma^{\prime}}{\Gamma}(1+i r) d r+n h(0) \\
& +\sum_{\{P\}} \sum_{k=1}^{\infty} \frac{\log N(P)}{N(P)^{k / 2}+N(P)^{-k / 2}} g(k \log N(P)) .
\end{aligned}
$$

The notation is as follows: $n=$ number of cusps; $\Phi(s)$ is the scattering matrix, appearing as a factor in the zero Fourier coefficient of the Eisenstein series, and $\varphi(s)$ is its determinant. The numbers $r_{j} \in(-\infty, \infty) \cup i\left[-\frac{1}{2}, \frac{1}{2}\right]$ correspond to the eigenvalues $r_{j}^{2}+\frac{1}{4}$ of $\Delta$ and appear with the multiplicities of these

Received by the editors April 25, 1990.

1991 Mathematics Subject Classification. Primary 30F35.

The work of the first author was supported in part by the NSF Grant DMS 89-03076 and that of the second author by NSF Grant DMS 84-51759. 
eigenvalues, 0 being counted with double multiplicity. Finally $\rho_{j}$ runs over all the poles and zeros of $\varphi(s)$ in $\operatorname{Re} s<\frac{1}{2}$, counted with the order of the poles or the negative of the order of the zero. The $\{P\}$ term on the right is the usual sum over the hyperbolic conjugacy classes.

Two things should be noted concerning the form of formula (1.1): (1) As $\Gamma$ varies in Teichmüller space the only term on the right that changes is the $\{P\}$ sum and this changes in a real analytic fashion on $T(\Gamma)$. (2) The sum on the left evaluates $h$ at points in the complex plane with nonnegative integral multiplicities. In fact since $\Phi\left(\frac{1}{2}\right)$ is real symmetric with $\Phi^{2}\left(\frac{1}{2}\right)=I$, the term $\left(n+\operatorname{tr}\left[\Phi\left(\frac{1}{2}\right)\right]\right) / 2$ is a nonnegative integer. The possible negative factor for $\left(h\left(i\left(\frac{1}{2}-\rho_{j}\right)\right)\right.$, arising from a zero (instead of a pole) of $\varphi$ at $\rho_{j}$, is countered by the $h\left(r_{j}\right)$ as follows: If $\rho_{j}$ is a zero of $\varphi$ of order $\nu_{j}$, then the dimension of the eigenvalue $\rho_{j}\left(1-\rho_{j}\right)$ (coming from a residue of the Eisenstein series at $\left.s=1-\rho_{j}\right)$ is $\geq \nu_{j}($ see $[\mathrm{Se}]$ or Theorem 4.1$)$.

With this motivation we define the singular set $\sigma(\Gamma)$ as follows: (i) If $\operatorname{Im} r \leq$ 0 and $r \neq 0$ we define the multiplicity of $r$, namely, $m(r)$, to be the dimension of the eigenspace for $\frac{1}{4}+r^{2}$ of $\Delta$ on $\Gamma \backslash H$; hence $m(r)=0$ unless $r \epsilon$ $(-\infty, \infty) \cup-i\left(0, \frac{1}{2}\right]$. (ii) If $\operatorname{Im} r>0$ then $m(r)$ is the multiplicity of the eigenvalue $\frac{1}{4}+r^{2}$ plus the order of the pole (or the negative of the order of the zero) of $\varphi(s)$ at $s=\frac{1}{2}+i r$, (iii) For $r=0$ we define $m(0)$ to be twice the multiplicity of cusp forms plus $\left(n+\operatorname{tr}\left[\Phi\left(\frac{1}{2}\right)\right]\right) / 2$. The singular set $\sigma(\Gamma)$ is then by definition the set of $r$ 's in $\mathbb{C}$ such that $m(r)>0$, counted with their multiplicities.

From the previous comments we see that $m(r) \geq 0$ and integer valued. Also if $\operatorname{Im} r>0$ and $r \notin i\left(0, \frac{1}{2}\right]$, then $m(r)$ is just the multiplicity of the pole of $\varphi(s)$ at $s=\frac{1}{2}+i r$, since in this case, $\varphi(s)$ has no zeros [LP1] and $\frac{1}{4}+r^{2}$ is not an eigenvalue. For $r \in \mathbb{R}, r \neq 0, m(r)$ is the dimension of the cusp forms with eigenvalue $\frac{1}{4}+r^{2}$; this follows from the fact that $\Phi(s)$ is regular (in fact unitary) on $\operatorname{Re} s=\frac{1}{2}$ and that any $L^{2}$ eigenfunction is a cusp form. Note that the left side of (1.1) may now be written as

$$
\sum_{r \in \sigma(\Gamma)} m(r) h(r)
$$

By a previous remark we see that (1.2) is real analytic in $T(\Gamma)$ for a fixed $h$.

One of our main results is the real analyticity in $T(\Gamma)$ of the singular set. We prove that as $\Gamma$ varies on a real analytic curve $\Gamma_{t}$ in $T\left(\Gamma_{0}\right), \sigma\left(\Gamma_{t}\right)$ has at most algebraic singularities. More precisely, if $r_{0}$ in $\sigma\left(\Gamma_{0}\right)$ has multiplicity $m\left(r_{0}\right)$, then there is an $\varepsilon>0$, a neighborhood $U$ of $r_{0}$, and a Puisseux series $r(t),|t|<\varepsilon$, with $m\left(r_{0}\right)$ branches such that $r(t) \in \sigma\left(\Gamma_{t}\right)$ for $|t|<\varepsilon$ and these $m\left(r_{0}\right)$ branches fill out $U \cap \sigma\left(\Gamma_{t}\right)$. If, in particular, $r_{0}$ is simple then $r(t)$ is analytic in $t$. For some related questions on the continuity of the spectrum of $\Gamma$ see Hejhal $[\mathrm{He}]$ and Venkov [V].

Another result, easily derived once the machinary and basic identifications 
have been set up, is an explicit formula for

$$
I=\left.\frac{d^{2}}{d t^{2}} \operatorname{Im} r_{j}(t)\right|_{t=0},
$$

where $0 \neq r_{j}(0) \in \mathbb{R}$ corresponds to a simple cusp form for $\left.\Gamma_{t}\right|_{t=0}$, see (5.29). A formula of this type is known in the physics literature as Fermi's Golden Rule (see Reed and Simon [RS]) and we will refer to it as such. That $I \neq 0$ implies the destruction of the cusp form at $r_{j}(0)$ is precisely the condition found in [PS1]. Wolpert [W] has recently given another derivation that $I \neq 0$ is both necessary and sufficient to first order for the destruction of a cusp form.

Our approach to this problem is via the automorphic wave equation, as developed by Lax-Phillips in [LP1, LP3], where the basic notions of incoming and outgoing subspaces, the semigroup $Z(t)$, and its infinitesimal generator $B$ come into play. These ideas are reviewed in $\S 2$. The operator $B$ is not selfadjoint but it does have a compact resolvent and hence a pure point spectrum. The basic technical results of the paper are developed in $\S \S 3$ and 4 . There it is proved that the spectrum of $B$ (with algebraic multiplicities) is precisely $\sqrt{-1}$ times the singular set introduced above. Once this is established, the results mentioned earlier follow easily from standard perturbation theory.

The mini-trace formula, proved in $\S 4$, connects the multiplicities of the eigenvalues of $B$ with the order of the poles of $\varphi$. In $\S 5$ the perturbation results are obtained, including the Puisseux expansion for the multiplicities and the Fermi-Golden Rule.

In $\S 6$ we show how to adapt the previous theory to include the $\chi$-twisted spectral problem, where $\chi$ is a unitary character of $\Gamma$. In particular, we obtain the analyticity properties for the smallest eigenvalue $\lambda_{0}(\chi)$ of the Laplacian that were claimed in our paper "Spectrum of Fermat curves" [PS2]. We also treat the dependence of the integer-valued function $\operatorname{tr}\left[\Phi\left(\frac{1}{2}, \Gamma, \chi\right)\right]$ on the parameter $\Gamma$ in $T(\Gamma)$ or on the character $\chi$. It seems likely that the generic value for this quantity is $-n$ (where $n$ is the number of singular cusps). We have shown this to be the case for the Teichmüller spaces of the once punctured torus $(n=1)$ and the six times punctured sphere $(n=6)$. Finally we construct an example $\{\Gamma, \chi\}$ (coming from the Fermat curve analysis) with one cusp for which $\Phi\left(\frac{1}{2}, \Gamma, \chi\right)$ is generically -1 in $\chi$ and equal to +1 on a nonempty discrete set.

\section{THE HYPERBOLIC WAVE EQUATION}

We shall limit ourselves to discrete subgroups of $\operatorname{PSL}(2, \mathbb{R})$ with noncompact fundamental domains $F$ of finite volume in the hyperbolic plane. Let $L_{0}$ denote a Laplacian on $F$. The hyperbolic wave equation for $L_{0}$ is of the form

$$
u_{t t}=L u=L_{0} u+u / 4
$$

with initial values $f=\left\{f_{1}, f_{2}\right\}$,

$$
u(z, 0)=f_{1}(z) \text { and } u_{t}(z, 0)=f_{2}(z) .
$$

The energy form for the wave equation is

$$
E(f)=-\left(f_{1}, L f_{1}\right)+\left(f_{2}, f_{2}\right),
$$


where parenthesis denotes the $L^{2}$ inner product

$$
(f, g)=\int_{F} f \bar{g} \frac{d x d y}{y^{2}} .
$$

An integration by parts brings $E$ into a more symmetric form,

$$
E(f)=\int_{F}\left(y^{2}\left|\partial f_{1}\right|^{2}-\frac{\left|f_{1}\right|^{2}}{4}+\left|f_{2}\right|^{2}\right) \frac{d x d y}{y^{2}},
$$

and it is evident from this that $E$ is in general not positive definite. To get around this problem we choose a partition of unity $\left[\phi_{j} ; j=0,1, \ldots, m\right]$ with $\phi_{0}$ of compact support and $\phi_{j}=1$ in the $j$ th cusp (transformed to $\infty$ ) for $y>a, a$ fixed. We then set

$$
\begin{gathered}
E_{j}(f)=\int \phi_{j}\left(y^{2}\left|\partial f_{1}\right|^{2}-\frac{\left|f_{2}\right|^{2}}{4}+\left|f_{2}\right|^{2}\right) \frac{d x d y}{y^{2}}, \\
E=\sum_{j=0}^{m} E_{j} .
\end{gathered}
$$

Another integration by parts allows us to write this as

$$
E_{j}(f)=\int \phi_{j}\left(y\left|\partial \frac{f_{1}}{y^{1 / 2}}\right|^{2}+\frac{\left|f_{2}\right|^{2}}{y^{2}}\right) d x d y-\int \partial_{y} \phi_{j} \frac{\left|f_{1}\right|^{2}}{2 y} d x d y
$$

It is clear from this that

$$
G(f)=E(f)+J(f)
$$

with

$$
J(f)=c \int_{M}\left|f_{1}\right|^{2} d x d y
$$

is pointwise positive definite when $c$ is sufficiently large and $M$ is some compact subset of $F$. It is not hard to show that $J$ is compact with respect to $G$ (see [LP1]).

We now define the Hilbert space $H_{G}$ as the completion with respect to $G$ of $C^{\infty}$ data with compact support. It can be shown that the $E$ and $G$ forms are equivalent on any closed subspace of $H_{G}$ on which $E$ is positive (see [LP1]).

Next we rewrite the wave equation in the form

$$
f_{t}=A f \text {, }
$$

where the infinitesimal generator $A$ is

$$
A=\left(\begin{array}{ll}
0 & I \\
L & 0
\end{array}\right),
$$

defined as the closure of $A$, restricted to $C_{0}^{\infty}(F) \times C_{0}^{\infty}(F)$. It is shown in [LP1] that $A$ generates a group of bounded operators $U(t)$ that grow exponentially in the $G$-norm but are unitary with respect to the energy form $E$.

Of particular interest to us are certain subspaces of $H_{G}$ that are called incoming and outgoing, denoted by $D_{-}$and $D_{+}$, respectively. We define $D_{-}$ 
and $D_{+}$to have support only in the cusps and in each cusp (transformed to $\infty)$ to be of the form

$$
\begin{aligned}
& D_{-}=\left\{y^{1 / 2} \phi(y), y^{3 / 2} \phi^{\prime}(y)\right\}, \\
& D_{+}=\left\{y^{1 / 2} \phi(y),-y^{3 / 2} \phi^{\prime}(y)\right\},
\end{aligned}
$$

where $\phi \in C^{\infty}$ vanishes for $y<a$ and $\phi^{\prime}=d \phi / d y$; $a$ is fixed but arbitrary. The corresponding solutions of (2.1) with incoming and outgoing initial data are

$$
u(z, t)=y^{1 / 2} \phi\left(y e^{t}\right) \text { and } u(z, t)=y^{1 / 2} \phi\left(y e^{-t}\right),
$$

respectively, at least while the support for these functions remains in the cusp.

It is clear from this that

$$
\begin{array}{ll}
U(t) D_{-} \subset D_{-} & \text {for } t \leq 0, \\
U(t) D_{+} \subset D_{+} & \text {for } t \geq 0,
\end{array}
$$

and

$$
\bigcap U(t) D_{-}=\{0\}=\bigcap U(t) D_{+} .
$$

It is clear from (2.7) that

$$
D_{-} \text {and } D_{+} \text {are } E \text {-orthogonal. }
$$

We now choose $M$ so that $\{y>a\} \cap M=\varnothing$, in which case $D_{-}$and $D_{+}$are also $G$-orthogonal. The relations (2.12)-(2.14) also hold for the closures of $D_{-}$ and $D_{+}$.

Let us suppose that a given cusp, transformed to $\infty$, is of the form $F_{c} \times\{y>$ $a$ ) and denote the zero Fourier coefficient of $v$ with respect to this cusp by $v^{(0)}$

$$
v^{(0)}(y)=\frac{1}{\sqrt{\left|F_{c}\right|}} \int_{F_{c}} v(x, y) d x .
$$

A necessary and sufficient condition for data $f=\left\{f_{1}, f_{2}\right\}$ in $H_{G}$ to be $E$ orthogonal to $D_{-}$is that the zero Fourier components of $f$ satisfy

$$
f_{2}^{(0)}=-y^{3 / 2} \partial_{y}\left(\frac{f_{1}^{(0)}}{y^{1 / 2}}\right) \text { for } y>a,
$$

in each cusp. If $f$ is merely locally in $H_{G}$ then $f_{2}^{(0)}$ can have an additional term of the type $c y^{1 / 2}$. Similarly $f$ in $H_{G}$ is $E$-orthogonal to $D_{+}$iff

$$
f_{2}^{(0)}=y^{3 / 2} \partial y\left(\frac{f_{1}^{(0)}}{y^{1 / 2}}\right) \text { for } y>a \text {. }
$$

If $f$ is $E$-orthogonal to both $D_{-}$and $D_{+}$then $(2.16)$ implies that

$$
f^{(0)}=\left\{c y^{1 / 2}, 0\right\} \text { for } y>a \text {. }
$$


We denote the set of all such data by $K$,

$$
K=H_{G} \ominus\left(D_{-} \oplus D_{+}\right) .
$$

The $E$-orthogonal (and $G$-orthogonal) projection onto $K$ is

$$
\begin{aligned}
& P f=f \quad \text { except for the zero coefficient in each cusp, } \\
& (P f)^{(0)}=\left\{f_{1}^{(0)}(a)(y / a)^{1 / 2}, 0\right\} \quad \text { for } y>a \text { in each cusp. }
\end{aligned}
$$

Finally we set

$$
Z(t)=P U(t) P \text { for } t \geq 0 .
$$

It can be shown (see [LP1, Theorem 2.7]) that the operators $Z$ form a strongly continuous semigroup of operators on $K$. We denote the infinitesimal generator of $Z$ by $B$. Since $U$ grows at most exponentially,

$$
\|Z(t)\| \leq C e^{\omega t}
$$

for some $\omega>0$. Hence for $f$ in $K$ and $\operatorname{Re} \lambda>\omega$ we have

$$
(\lambda I-B)^{-1} f=\int_{0}^{\infty} e^{-\lambda t} Z(t) f d t=P \int_{0}^{\infty} e^{-\lambda t} U(t) f d t=P(\lambda I-A)^{-1} f
$$

It can be shown (see [LP1] or [LP3]) that the resolvent of $B$, denoted by $R_{\lambda}(B)$, is compact, and it follows that $B$ has a pure point spectrum of finite multiplicity and that $R_{\lambda}(B)$ is meromorphic in the entire complex plane.

We see from (2.12) that for $f$ orthogonal to $D_{-}$and $t \geq 0$

$$
Z(t) P f=P U(t) P f=P U(t) f .
$$

If $f$ also belongs to $D(A)$ then

$$
B P f=P A f \text {. }
$$

Before concluding this section we introduce the time reversal operator

$$
R\left\{f_{1}, f_{2}\right\}=\left\{f_{1},-f_{2}\right\} .
$$

It is readily verified that

$$
U(-t)=R U(t) R
$$

and it follows from this that

$$
R A R=-A=A^{*}, \quad R B R=B^{*}, \quad R D_{-}=D_{+}, \quad R D_{+}=D_{-} .
$$

\section{THE SPECTRUM OF $B$}

Next we characterize the spectrum of $B$. The nonzero eigenvalues of $B$ can be related to those of $A$. To this end we say that a data $f$ is an outgoing eigenfunction of $A$ with eigenvalue $\lambda(1)$ if $(A-\lambda)^{k} f=0$ pointwise for some $k,(2)$ if $f$ is outgoing, that is, satisfies $(2.16)_{-}$, and (3) if $A^{j} f$ minus its zero Fourier coefficient in the cusps lies in $H_{G}$ for $j=0,1, \ldots, k-1 . \lambda$ is called a scattering frequency if some $\lambda$-outgoing eigenfunction $f$ is not in $H_{G}$. 
The scattering frequencies correspond to poles of the scattering matrix in $\operatorname{Re} \lambda<0$. Indeed for the Eisenstein series data $\left\{e_{\lambda}, \lambda e_{\lambda}\right\}$, it is only the residues which are outgoing.

We call the set of all (outgoing) data $f$ for which $(A-\lambda)^{k} f=0$ for some $k$, the $\lambda$-eigenspace of $A$; the dimension of the eigenspace is called the multiplicity of $\lambda$. An eigenvalue of $A$ is simple if $A$ is diagonalizable in its eigenspace.

A few remarks are in order. We shall show later on that when $f$ is an outgoing eigenfunction of $A$ and $\operatorname{Re} \lambda>0$, then $f$ lies in $H_{G}$. When $\operatorname{Re} \lambda=$ $0, \lambda \neq 0$, there are exactly $n$ linearly independent Eisenstein series, no linear combination of which is outgoing. It therefore follows from Maass's theorem $[\mathrm{M}]$ that, modulo the Eisenstein series, every other eigenfunction is a cusp form and therefore belongs to $H_{G}$. Hence when $\operatorname{Re} \lambda \geq 0, \lambda \neq 0$, all of the outgoing eigenfunctions are eigenfunctions of the selfadjoint Laplacian.

Suppose $A f=\lambda f$; then

$$
f_{2}=\lambda f_{1} \text { and } L f_{1}=\lambda f_{2} \text { so that } L f_{1}-\lambda^{2} f_{1}=0 \text {. }
$$

If $f$ belongs to $H_{G}$ then $f_{2}$ is square integrable, and if, in addition, $\lambda \neq 0$ then $f_{1}$ is also. Hence $f_{1}$ is an eigenfunction of $L$ when considered as a selfadjoint operator on $L^{2}(F)$, and if $\operatorname{Re} \lambda>0$ then $\lambda \in\left(0, \frac{1}{2}\right]$. If $\operatorname{Re} \lambda<0$ and $f_{1}^{(0)} \neq 0$ in some cusp, then the outgoing condition implies that $f_{1}$ is not in $L^{2}(F)$. Consequently if $\lambda \neq 0$, the only outgoing bound states for $A$ have $\operatorname{Re} \lambda \geq 0$ unless $f$ is a cusp form, in which case $\left\{f_{1},-\lambda f_{1}\right\}$ is also a bound state at $-\lambda$. It also follows from the above for $\operatorname{Re} \lambda \geq 0, \lambda \neq 0$, that $\lambda$ is simple. For suppose that $(A-\lambda)^{2} f=0$. Setting $g=(A-\lambda) f$; since $f$, $g \in H_{G}$ and $(A-\lambda) g=0$, we then conclude as before that $g_{2}=\lambda g_{1} \in L^{2}$, $L g_{1}-\lambda^{2} g_{1}=0$, and $f_{1}=\left(f_{2}-g_{1}\right) / \lambda \in L^{2}$. But then

$$
L f_{1}-\lambda^{2} f_{1}=2 \lambda g_{1},
$$

which has no $L^{2}$ solution.

The relation (2.24) suggests that the action of $P$ on an outgoing eigenfunction of $A$ produces an eigenfunction of $B$; we shall see in (3.17) that this is true for $\lambda \neq 0$. When $\operatorname{Re} \lambda \neq 0$ it is clear from the form of the zero Fourier coefficient that one can obtain an outgoing eigenfunction of $A$ from an Eisenstein series only as the residue at a pole, and conversely each such residue is an outgoing eigenfunction of $A$.

Next suppose that $(B-\lambda)^{k} f=0$ for some $k \geq 1$. Then

$$
Z(t) f=e^{\lambda t} \exp ((B-\lambda) t) f=e^{\lambda t} \sum_{j=0}^{k-1} \frac{t^{j}}{j !}(B-\lambda)^{j} f .
$$

We shall prove by induction that $f$ is 'outgoing' in each cusp in the sense that

$$
f_{2}^{(0)}(y)=-y^{3 / 2} \partial_{y}\left(\frac{f_{1}^{(0)}}{y^{1 / 2}}\right) \text { for } y<a
$$

and that

$$
(Z(t) f)_{1}^{(0)}=f_{1}^{(0)}\left(y e^{-t}\right) \text { for } a e^{-t}<y<a
$$


In order to include the case $k=1$ in the induction argument we note that the result is trivially true for $k=0$. Suppose that it holds for $k-1$ and set $(B-\lambda) f=g$. Then

$$
\begin{aligned}
Z(t) f & =e^{\lambda t} f+e^{\lambda t} \sum_{j=0}^{k-2} \frac{t^{j+1}}{(j+1) !}(B-\lambda)^{j} g \\
& =e^{\lambda t} f+e^{\lambda t} \int_{0}^{t} e^{-\lambda \tau} Z(\tau) g d \tau .
\end{aligned}
$$

Our strategy is to compare this expression with

$$
(Z(t) f)_{1}^{(0)}=(P U(t) f)_{1}^{(0)},
$$

which is equal to $z(y, t)=(U(t) f)_{1}^{(0)}$ in the region $a e^{-t}<y<a$.

It is convenient to make the substitutions

$$
w=z / y^{1 / 2} \text { and } s=\log y,
$$

after which the equation for $w(s, t)$ is simply

$$
w_{t t}=w_{s s}
$$

with initial data

$$
w(s, 0)=u(s) \text { and } \quad w_{t}(s, 0)=v(s)
$$

where $\{u, v\}=f / y^{1 / 2}$.

The relation $(B-\lambda) f=g$ becomes

$$
v-\lambda u=h_{1} \quad \text { and } \quad L u-\lambda v=h_{2}, \quad \text { so that } L u-\lambda^{2} u=\lambda h_{1}+h_{2},
$$

where $h=g / y^{1 / 2}$ and the assumptions (3.4) and (3.5) on $g$ translate into

$$
\begin{gathered}
h_{2}(s)=-\partial_{s} h_{1}(s) \quad \text { for } s<\alpha=\log a \\
(Z(t) g)_{1}^{(0)}(y) \rightarrow h_{1}(s-t) \quad \text { for } \alpha-t<s<\alpha .
\end{gathered}
$$

It is easy to check that a particular solution for $L u-\lambda^{2} u=\lambda h_{1}-\partial_{s} h_{1}$ is

$$
u_{0}(s)=-e^{-\lambda s} \int_{0}^{s} e^{\lambda \sigma} h_{1}(\sigma) d \sigma .
$$

Hence $u$ itself must be of the form

$$
u(s)= \begin{cases}b e^{-\lambda s}+c e^{\lambda s}+u_{0}(s) & \text { for } s \leq \alpha, \\ b e^{-\lambda \alpha}+c e^{\lambda \alpha}+u_{0}(\alpha) & \text { for } s>\alpha\end{cases}
$$

and $v=\lambda u+h_{1}$ for $s \leq \alpha$ and $v=0$ for $s>\alpha$. Notice that $u_{0}^{\prime}=-\lambda u_{0}-h_{1}$.

The solution of (3.8) is of the form

$$
w(s, t)=\phi(s-t)+\psi(s+t)
$$

where

$$
\phi(s)+\psi(s)=u(s) \quad \text { and } \quad-\phi^{\prime}(s)+\psi^{\prime}(s)=v(s) .
$$


A straightforward calculation shows that

$$
\begin{aligned}
& \phi(s)=u(s)-c e^{\lambda s}+e_{1}, \psi(s)=c e^{\lambda s}+e_{2} \text { for } s \leq \alpha, \\
& \phi(s)=\phi(\alpha), \psi(s)=\psi(\alpha) \text { for } s>\alpha, e_{1}+e_{2}=0
\end{aligned}
$$

Substituting this into (3.11) we find that for $\alpha-t<s<\alpha$

$$
w(s, t)=b e^{-\lambda(s-t)}+c e^{\lambda \alpha}-e^{-\lambda(s-t)} \int_{0}^{s-t} e^{\lambda \sigma} h_{1}(\sigma) d \sigma .
$$

On the other hand we see from $\left(3.5^{\prime}\right)$ and (3.6) that in this same range

$$
\begin{aligned}
(Z(t) f)_{1}^{(0)} & \rightarrow e^{\lambda t}\left[u(s)+\int_{0}^{t} e^{-\lambda \tau} h_{1}(s-\tau) d \tau\right] \\
& =e^{\lambda t}\left[b e^{-\lambda s}+c e^{\lambda s}-e^{-\lambda s} \int_{0}^{s-t} e^{\lambda \sigma} h_{1}(\sigma) d \sigma\right] .
\end{aligned}
$$

The expressions (3.13) and (3.14) are equal only if $c=0$. In this case

$$
\begin{gathered}
u=b e^{-\lambda s}+u_{0}(s) \text { and } v=\lambda u+h_{1}=-\partial_{s} u(s) \text { for } s \leq \alpha, \\
w(s, t)=u(s-t) \text { for } \alpha-t<s<\alpha .
\end{gathered}
$$

This translates into (3.4) and (3.5) for $Z(t) f$ and so completes the induction.

For $s \leq \alpha$ in the above situation, $v=-\partial_{s} u$ and (by induction from (3.10) and (3.15)) we see that $u, v$ are sums of terms of the sort $s^{j} e^{-\lambda s}$. By continuing $u$ and $v$ to be equal to these same sums for $s>\alpha$ the relation $v=-\partial_{s} u$ persists. The data $f_{e}$, with zero Fourier coefficient equal to the translate of the so-extended $u$ and $v$, becomes an outgoing data satisfying $(A-\lambda)^{k} f_{e}=0$ and if $\operatorname{Re} \lambda>0$ then $f_{e}$ is in $H_{G}$.

Because of the finite speed of propagation for solutions of the wave equation, it is easy to see for data $f$, which is locally in $H_{G}$, that $U(t) f$ is well defined. If $f$ is outgoing then it follows from (2.12) and (2.16) _ that $Z(t) f=P U(t) P f=$ $P U(t) f$. Finally if $f$ is an outgoing eigenfunction, i.e., $(A-\lambda)^{k} f=0$, then by differentiating in the local $H_{G}$ topology we get

$$
\frac{d^{j}}{d t^{j}} e^{-\lambda t} U(t) f=e^{-\lambda t}(A-\lambda)^{j} U(t) f=e^{-\lambda t} U(t)(A-\lambda)^{j} f,
$$

and from the Taylor series expansion for $e^{-\lambda t} U(t) f$ we obtain

$$
U(t) f=e^{\lambda t} \sum_{j=0}^{k-1} \frac{t^{j}}{j !}(A-\lambda)^{j} f .
$$

All of the expressions $P(A-\lambda)^{j} f$ are in $H_{G}$ so that

$$
(B-\lambda)^{k} P f=\left.\frac{d^{k}}{d t^{k}} e^{-\lambda t} Z(t) f\right|_{t=0}=\left.\frac{d^{k}}{d t^{k}} e^{-\lambda t} P U(t) f\right|_{t=0}=0 .
$$

If $f$ is outgoing and $(A-\lambda) f=0$, it follows from a theorem of Maass [M] that $f$ is either an Eisenstein series (in which case $\lambda$ corresponds to a pole of the scattering matrix and hence is a scattering frequency if $\operatorname{Re} \lambda<0$ or in $H_{G}$ if $\operatorname{Re} \lambda>0$ ) or a cusp form. We summarize these results with 
Theorem 3.1. Suppose $\lambda, \lambda \neq 0$, is an eigenvalue of $B$ or an outgoing eigenvalue of $A$, then the multiplicity of $B$ at $\lambda$ is the same as that of the outgoing eigenspace of $A$. If $\operatorname{Re} \lambda<0$ then $\lambda$ is a scattering frequency unless the corresponding eigenspace has only cusp forms, which can only happen when $\lambda \in\left[\frac{-1}{2}, 0\right)$. If $\operatorname{Re} \lambda=0$ then the eigenspace is simple and filled with cusp forms. If $\operatorname{Re} \lambda>0$, i.e., if $\lambda \in\left(0, \frac{1}{2}\right]$, then the eigenspace of $B$ at $\lambda$ contains only data in $H_{G}$, it is simple, and its multiplicity is the same as that of $L$ at $\lambda^{2}$. The time reversal operator $R$ sets up a one-to-one correspondence between the cusp form eigendata at $\lambda$ and those at $-\lambda$.

Next we consider the null eigenspace of $B$, that is, the set of data $f$ such that $B^{k} f=0$ for some $k$. If $\theta$ is a cusp form eigenfunction of $L$, i.e., $L \theta=0$, then $B\{\theta, 0\}=0$ and $B^{2}\{0, \theta\}=0$. Moreover if $e(s), s=\lambda+\frac{1}{2}$, is a nonzero Eisenstein series at $s=\frac{1}{2}$ (and hence having its zero Fourier coefficient in cusps of the form $b y^{1 / 2}$ ), then $B\{e, 0\}=0$. Since the scattering matrix $\Phi$ at $s=\frac{1}{2}$ is unitary with $\Phi^{2}=I$, there will be exactly $\operatorname{tr}[\Phi+I] / 2$ linearly independent Eisenstein series of this type. We now show that these are the only data in the null eigenspace of $B$. (The discussion of the null eigenspace of $B$ in [LP1] is not correct.)

Theorem 3.2. $B^{k} f=0$ iff $f$ can be extended to an outgoing eigenfunction $f_{e}$ of $A$ with $A^{k} f_{e}=0 ; k=1$ unless $f_{2}$ is a cusp form, in which case $k=2$. The dimension of the null eigenspace of $B$ is equal to $p+2 q$, where $p=\operatorname{tr}\left[\Phi\left(\frac{1}{2}\right)+I\right] / 2$ and $q$ is the number of linearly independent cusp form eigenfunctions of $L$.

Proof. Suppose that $B^{k} f=0$ so that

$$
Z(t) f=\exp (t B) f=\sum_{j=0}^{k-1} \frac{t^{j}}{j !} B^{j} f .
$$

Next we compute $(Z(t) f)^{(0)}$ from the expression $Z(t) f=P U(t) P f$ and compare the result with (3.18). As before we let $z(y, t)=(U(t) f)_{1}^{(0)}$. Set $m=k / 2$ if $k$ is even and $m=(k+1) / 2$ if $k$ is odd. Then $B^{2 m} f=0$ and consequently $L^{m} f_{1}=0=L^{m} f_{2}$ for $y<a$ in the cusps of $F$. Again we make the substitution $w=z / y^{1 / 2}$,

$$
u=w(s, 0)=f_{1} / y^{1 / 2}, \quad v-w_{t}(s, 0)=f_{2} / y^{1 / 2}, \quad s=\log y .
$$

Then the conditions $L^{m} f_{1}=0=L^{m} f_{2}$ for $y<a$ can be rewritten as

$$
\partial_{s}^{2 m} u=0=\partial_{s}^{2 m} v \text { for } s<\alpha=\log a .
$$

This means that

$$
u(s)=\sum_{j=0}^{2 m-1} a_{j} s^{j} \quad \text { and } \quad v(s)=\sum_{j=1}^{2 m} j b_{j} s^{j-1}
$$


for $s<\alpha$ and, since $f$ belongs to $K$, that

$$
u(s)=\sum_{j=0}^{2 m-1} a_{j} \alpha^{j} \text { and } v(s)=0
$$

for $s>\alpha$. Using these expressions as initial conditions for the classical wave equation $w_{t t}=w_{s s}$, we now obtain an explicit expression for $w(s, t)$ in the region $\alpha-t<s<\alpha$, where $Z(t) f$ and $U(t) f$ should coincide.

Again we write the solution as

$$
w(s, t)=\varphi(s-t)+\psi(s+t),
$$

where

$$
u(s)=\varphi(s)+\psi(s) \text { and } v(s)=-\varphi^{\prime}(s)+\psi^{\prime}(s) .
$$

One finds that

$$
\begin{aligned}
& \varphi(s)=\left(u(s)-\int_{\alpha}^{s} v(\sigma) d \sigma\right) / 2+e_{1}, \\
& \psi(s)=\left(u(s)+\int_{\alpha}^{s} v(\sigma) d \sigma\right) / 2+e_{2},
\end{aligned}
$$

with $e_{1}+e_{2}=0$. Replacing $u$ and $v$ by (3.21) and substituting these values into (3.22), we get for $\alpha-t<s<\alpha$

$$
\begin{gathered}
w(s, t)=\left(\sum_{j=0}^{2 m-1} a_{j}(s-t)^{j}-\sum_{j=1}^{2 m} b_{j}(s-t)^{j}\right) / 2+c, \\
\text { where } c=\left(u(\alpha)+\sum_{j=1}^{2 m} b_{j} \alpha^{j}\right) / 2 .
\end{gathered}
$$

Comparing the coefficient of $t^{0}$ in this expression with that obtained from (3.18), namely, $\{u(s), v(s)\}$, we have

$$
\sum_{j=0}^{2 m-1} a_{j} s^{j}=u(s)=\left(\sum_{j=0}^{2 m-1} a_{j} s^{j}-\sum_{j=1}^{2 m} b_{j} s^{j}\right) / 2+c .
$$

This requires that $b_{j}=-a_{j}$ for $1 \leq j \leq 2 m-1$ and $b_{2 m}=0$. It follows from this that for $s<\alpha$

$$
w(s, t)=\sum_{j=0}^{2 m-1} a_{j}(s-t)^{j} \quad \text { and } \quad v=-\partial_{s} u .
$$

As in the proof of Theorem 3.1 this implies that $f$ can be extended to be an outgoing eigenfunction $f_{e}$ of $A$ with $A^{k} f_{e}=0$ and conversely.

Comparing the powers of $t$ in (3.18) and (3.27) we see that $a_{j}=0$ when $j \geq k$. Moreover if $k>2$ and $g=B^{k-1} f \neq 0$ then $a_{k-1} \neq 0$ in at least one of the cusps. For suppose that $a_{k-1}=0$ in all cusps. Then $g^{(0)}=0$ and hence $g_{1}=\theta$, a cusp form, and $g_{2}=0$. Setting $h=B^{k-3} f$ we see that $L h_{1}=\theta$. 
Following Maass we integrate by parts over a cut-off domain $F_{a}$, i.e., a domain cut-off in the cusps at $y=a$ only for the zero Fourier coefficient:

$$
\begin{aligned}
\int_{F}|\theta|^{2} d x d y & =\int_{F_{a}}\left(L h_{1} \bar{\theta}-h_{1} \overline{L \theta}\right) \frac{d x d y}{y^{2}} \\
& =\left.\sum_{\text {cusps }}\left(\partial_{y} h_{1}^{(0)} \overline{\theta^{(0)}}-h_{1}^{(0)} \overline{\partial_{y} \theta^{(0)}}\right)\right|_{y=a}=0,
\end{aligned}
$$

which is impossible. Finally if $k=2$ and $a_{1}=0$ in all of the cusps then $f_{2}^{(0)}=0$ in all of the cusps and $f_{2}$ is a cusp form.

Next suppose that $k>1$ and when $k=2$ that $f_{2}$ is not a cusp form. Then $b=a_{k-1} \neq 0$ in at least one of the cusps. Set $g=B^{k-1} f$ and $h=B^{k-2} f$. Then $L h_{1}=g_{2}=0=L g_{1}$ and it follows from (3.27) that in the $i$ th cusp

$$
h_{1}^{(0)}=y^{1 / 2}\left(c_{i}+b_{i} \log y\right) \text { and } g_{1}^{(0)}=b_{i} y^{1 / 2} \text {. }
$$

Again following Maass we have

$$
0=\int_{F_{a}}\left(L h_{1} \overline{g_{1}}-h_{1} \overline{L g_{1}}\right) \frac{d x d y}{y^{2}}=\sum\left|b_{i}\right|^{2},
$$

which is again impossible. Hence if $B^{k} f=0$ then, in fact, $B f=0$ unless $k=2$ and $f_{2}$ is a cusp form.

According to Maass's theorem all of the noncuspidal generalized eigenfunctions of $L_{0}$ at $s=\frac{1}{2}$ (i.e., $\lambda=0$ ) are either Eisenstein series or derivatives (with respect to $s$ ) thereof. If the scattering matrix has $p$ positive eigenvalues (and $n-p$ negative eigenvalues) then there will be exactly $p$ nonzero linearly independent Eisenstein series at $s=\frac{1}{2}$ and these will have zero Fourier coefficient $b y^{1 / 2}$. There will also be $n-p$ linear combinations of Eisenstein series that vanish but have first derivatives with zero Fourier coefficients of the form $b y^{1 / 2}+c y^{1 / 2} \log y$. Such a derivative is again a null function of $L$ since from

$$
L_{0} e(s)+s(1-s) e(s)=0
$$

we get

$$
L_{0} e(s)^{\prime}+(1-2 s) e(s)+s(1-s) e(s)^{\prime}=0
$$

and when $s=\frac{1}{2}$ this becomes

$$
L_{0} e\left(\frac{1}{2}\right)^{\prime}+\frac{e(1 / 2)^{\prime}}{4}=0 .
$$

Notice that $f=\left\{e\left(\frac{1}{2}\right)^{\prime}, 0\right\}$ is not an outgoing eigendata of $A$. Thus from the $n$ Eisenstein contributions to the null space of $L$, there are only $p$ that contribute to the null eigenspace of $B$. This concludes the proof of Theorem 3.2 .

Remark 3.3. The zero Fourier coefficient of an eigenfunction of $A$ in a cusp is real analytic in $y$, and hence if the outgoing condition $(2.16)_{-}$is satisfied in any interval it will be satisfied for all $y>a$. This shows that the outgoing condition does not depend on a particular choice of $a$. It therefore follows 
from Theorem 3.1 and 3.2 that the spectrum of $B$ is also independent of the choice of $a$.

We shall eventually prove that

$$
\text { Spectrum of } B=i \sigma(\Gamma) \text {. }
$$

It follows from Theorems 3.1 and 3.2 that

Corollary 3.4. If $\operatorname{Im} r \leq 0$ then $m(r)=$ multiplicity of the eigenvalue $\lambda=$ ir of $B$.

Proof. If $r=0$ this is immediate from Theorem 3.2. Theorem 3.1 equates the eigendata of $B$ with the outgoing eigendata of $A$. If $\operatorname{Re} \lambda=0, \lambda \neq 0$, then the outgoing $\lambda$-eigenspace of $A$ is simple and filled with cusp forms of $L$ at $r^{2}, r=-i \lambda$. If $\lambda \in\left(0, \frac{1}{2}\right]$ then the outgoing eigenspace of $A$ is again simple and its multiplicity is the same as that of $L$ at $-\lambda^{2}$, i.e., $r$ in $i\left(0,-\frac{1}{2}\right]$.

\section{A MINI-TRACE FORMULA}

We have seen in the previous section that the scattering frequencies of $B$ occur at the poles of the scattering matrix. In this section we shall obtain a quantitative relation between the two. It will be convenient to express the scattering matrix $\Phi$ as a function of $\lambda$ rather than the usual parameter $s=$ $\lambda+\frac{1}{2}$. $\Phi$ is meromorphic with poles at the noncuspidal eigenvalues of $B$ off the imaginary axis. Denoting the determinant of $\Phi$ by $\varphi$ and setting $m(\lambda)$ equal to the multiplicity (i.e., the dimension of the eigenspace) of the eigenvalue $\lambda$ of $B$, we have

Theorem 4.1. For an eigenvalue $\lambda$ of $B$ off the imaginary axis, the order of the pole of $\varphi$ is equal to $m(\lambda)$ except when $\lambda \in\left[-\frac{1}{2}, 0\right)$ or $\left(0, \frac{1}{2}\right]$ in which case it is equal to $[m(\lambda)-m(-\lambda)]$.

The eigenspace for $L$ at $r$ and $-r$ are the same and correspond to that of $B$ at $|r|$. Hence combining Theorems 3.1 and 4.1 we have

Corollary 4.2. If $\operatorname{Im} r>0$, i.e., $\operatorname{Re} \lambda<0$, then $m(r)($ for $\sigma(\Gamma))=$ multiplicity of the eigenvalue $\lambda=$ ir of $B$.

Our proof of Theorem 4.1 is based on a mini-trace formula, which unfortunately requires the introduction of several new concepts. To begin with let $C_{0}^{(2)}[0, \infty)$ denote the set of $C^{(2)}$ functions $\theta$ on $[0, \infty)$ that vanish at 0 and for which

$$
|\theta|_{2}=\max \left[\int_{0}^{\infty} e^{\omega^{\prime} t}\left|\theta^{(i)}(t)\right| d t, \quad i=0,1,2\right]<\infty,
$$

where $\omega^{\prime}>\omega$ as defined in (2.21), and let

$$
Z(\theta)=\int_{0}^{\infty} \theta(t) Z(t) d t
$$

We note that

$$
Z\left(\theta_{1}\right) Z\left(\theta_{2}\right)=Z\left(\theta_{1} * \theta_{2}\right)
$$


Lemma 4.3. For $\theta$ in $C_{0}^{(2)}[0, \infty), Z(\theta)$ is of Hilbert Schmidt norm

$$
\|Z(\theta)\|_{2} \leq c|\theta|_{2} .
$$

We shall postpone the proof of this lemma until the end of this section. An obvious consequence of this result is

Corollary 4.4. For $\theta_{1}, \theta_{2}$ in $C_{0}^{(2)}[0, \infty), Z\left(\theta_{1} * \theta_{2}\right)$ is of trace class.

We denote the Fourier transform of $\theta$ by

$$
\hat{\theta}(\sigma)=\int_{0}^{\infty} e^{i \sigma t} \theta(t) d t
$$

It is easy to see that $\hat{\theta}(\sigma)=O\left(1 /\left|\sigma^{2}\right|\right)$.

The mini-trace formula deals with a $Z$-like semigroup of operators of the kind described in Chapter 3 of [LP1]. In order to orient the reader we shall give a brief description of the setup. Let $\left[q_{i}, i=1, \ldots, m\right]$ be the normalized eigenfunctions of $L$ with positive eigenvalues $\left[\lambda_{i}^{2}\right]$;

$$
L q_{i}=\lambda_{i}^{2} q_{i}, \quad \lambda_{i}>0 .
$$

We set $f_{i}^{ \pm}=\left\{q_{i}, \pm \lambda_{i} q_{i}\right\}$. These data belong to $H_{G}$ and are eigendata of $A$,

$$
A f_{i}^{ \pm}= \pm \lambda_{i} f_{i}^{ \pm}
$$

Let

$$
\mathscr{P}=\operatorname{span}\left[f_{i}^{ \pm}, i=1, \ldots, m\right]
$$

and set $H^{\prime}=E$-orthogonal complement of $\mathscr{P}$ in $H_{G}$. It is easy to see from (2.3) that $E$ is nonnegative on $H^{\prime}$. Finally we set $H_{E}^{\prime}$ equal to $H^{\prime}$ modulo the null set of $E$ in $H^{\prime}$. It can be shown that $H_{E}^{\prime}$ is complete in the $E$-norm and that $E$ and $G$ define equivalent norms on $H_{E}^{\prime}$. The operators $U(t)$ now defines a group of unitary operators on $H_{E}^{\prime}$, which is decomposed into the space spanned by the eigendata of $A$ (contained in $H_{E}^{\prime}$ ) and its orthogonal complement $H_{c}^{\prime}$. If $Q^{\prime}$ denotes the $E$-orthogonal projection of $H_{G}$ onto $H_{c}^{\prime}$, then it is easy to see that $Q^{\prime}$ commutes with $U$.

Next we define two sets of incoming and outgoing subspaces in $H_{E}^{\prime}$, namely,

$$
D_{ \pm}^{\prime}=Q^{\prime} \bar{D}_{ \pm} \quad \text { and } \quad D_{ \pm}^{\prime \prime}=\bar{D}_{ \pm} \cap H_{E}^{\prime}
$$

where $\bar{D}$ denotes the closure of $D$. It can be shown that

$$
D_{ \pm}^{\prime \prime} \subset D_{ \pm}^{\prime} \subset H_{c}^{\prime} \text {. }
$$

It is easy to verify that the analogues of (2.12) and $(2.12)^{\prime}$ hold for these subspaces. Instead of (2.14) we now have

$$
D_{+}^{\prime \prime} \perp D_{-}^{\prime} \quad \text { and } D_{+}^{\prime \prime} \perp D_{+}^{\prime \perp} \text {. }
$$

Next we make the further assumption that

$$
\overline{\bigcup U(t) D_{ \pm}^{\prime \prime}}=H_{c}^{\prime}
$$


and it follows that

$$
\overline{\bigcup U(t) D_{ \pm}^{\prime}}=H_{c}^{\prime}
$$

It is part of the general theory (see [LP1, Chapter 2]) that any subspace satisfying the analogues of $(2.12),(2.12)^{\prime}$, and (4.12) defines a translation representation

$$
f \in H_{c}^{\prime} \rightarrow k \in L^{2}\left(R, C^{n}\right)
$$

with the property that

$$
U(t) f \rightarrow k(s-t)
$$

We can also arrange it so that

$$
D_{-}^{\prime}, D_{-}^{\prime \prime} \rightarrow L^{2}\left(R_{-}, C^{n}\right) \text { and } D_{+}^{\prime}, D_{+}^{\prime \prime} \rightarrow L^{2}\left(R_{+}, C^{n}\right)
$$

We denote these various representators by $k_{-}^{\prime}, k_{-}^{\prime \prime}, k_{+}^{\prime}$, and $k_{+}^{\prime \prime}$, respectively.

The scattering operator $S^{\prime}$ is defined as the mapping

$$
S^{\prime}: k_{-}^{\prime} \rightarrow k_{+}^{\prime} \text {. }
$$

It is clear that $S^{\prime}$ is unitary and commutes with translation. In the spectral representation (obtained by taking the Fourier transform of the translation representation), the scattering operator takes the form of a multiplicative operator $\Phi(i \sigma)$ on $L^{2}\left(R, C^{n}\right)$, called the scattering matrix. To understand how $\Phi$ appears as a coefficient in the Eisenstein series, the reader is referred to Chapter 7 of [LP1], or better Theorem 6.5 of [LP3].

From this description of the scattering operator it is easy to factor $S^{\prime}$ as

$$
S^{\prime}=S_{+}^{-1} S_{0}
$$

where

$$
S_{0}: k_{-}^{\prime} \rightarrow k_{+}^{\prime \prime} \text { and } S_{+}: k_{+}^{\prime} \rightarrow k_{+}^{\prime \prime}
$$

Because of (4.11) these scattering operators have the additional property of being causal in the sense that they map $L^{2}\left(R_{-}, C^{n}\right)$ into itself. In the case of $k_{+}^{\prime}, L^{2}\left(R_{-}, C^{n}\right)$ corresponds to $D_{+}^{\prime \perp}$, which has the same properties as an incoming subspace. It follows from causality that the spectral representators of $\Phi_{0}$ and $\Phi_{+}$are inner factors.

We can associate $Z$-type semigroups with orthogonal pairs of incoming and outgoing subspaces

$$
Z_{0}(t)=P_{+}^{\prime \prime} U(t) P_{-}^{\prime} \quad \text { and } \quad Z_{+}(t)=P_{+}^{\prime \prime} U(t)\left(I-P_{+}^{\prime}\right)
$$

here $P$ 's are the orthogonal projections onto the orthogonal complements in $H_{c}^{\prime}$ of the associated subspaces, i.e., $P_{+}^{\prime}$ projects onto $H_{c}^{\prime} \ominus D_{+}^{\prime}$, etc., $Z_{0}$ acts on $K_{0}=H_{c}^{\prime} \ominus\left(D_{-}^{\prime} \oplus D_{+}^{\prime \prime}\right)$, and $Z_{+}$acts on $K_{+}=D_{+}^{\prime} \ominus D_{+}^{\prime \prime}$. 
Lemma 4.5. For $\theta_{1}, \theta_{2}$ in $C_{0}^{(2)}[0, \infty), Z_{0}\left(\theta_{1} * \theta_{2}\right)$ and $Z_{+}\left(\theta_{1} * \theta_{2}\right)$ are of trace class.

Proof. The dimension of $K_{+}\left(=\right.$the range of $\left.\left(P_{+}^{\prime \prime}-P_{+}^{\prime}\right)\right)$ is less than or equal to $m$, and so $Z_{+}(\theta)$ is obviously of trace class. On the other hand $D_{ \pm}^{\prime}=Q^{\prime} D_{ \pm}$ so that $P_{ \pm}^{\prime} Q^{\prime}\left(I-P_{ \pm}\right)=0$, and hence $\left(I-P_{ \pm}\right) Q^{\prime} P_{ \pm}^{\prime}=0$. Further $P_{ \pm}^{\prime} Q^{\prime}=$ $Q^{\prime} P_{ \pm}^{\prime}=P_{ \pm}^{\prime}$ and hence $P_{ \pm}^{\prime}=P_{ \pm}^{\prime} P_{ \pm}=P_{ \pm} P_{ \pm}^{\prime}$. We can therefore write

$$
\begin{aligned}
Z_{0} & =\left(P_{+}^{\prime \prime}-P_{+}^{\prime}\right) U(t) P_{-}^{\prime}+P_{+}^{\prime} U(t) P_{-}^{\prime} \\
& =\left(P_{+}^{\prime \prime}-P_{+}^{\prime}\right) U(t) P_{-}^{\prime}+P_{+}^{\prime} Z(t) P_{-}^{\prime} .
\end{aligned}
$$

It follows from this and Corollary 4.4 that $Z_{0}\left(\theta_{1} * \theta_{2}\right)$ is of trace class when $\theta_{1}, \theta_{2}$ belong to $C_{0}^{(2)}[0, \infty)$ since it can be expressed as the sum of two trace class operators.

Theorem 4.6. For $\theta_{1}, \theta_{2}$ in $C_{0}^{(2)}[0, \infty)$ and $Z^{0}=Z_{0}$ or $Z_{+}, \varphi^{0}=\varphi_{0}$ or $\varphi_{+}$, we have

$$
\operatorname{tr}\left[Z^{0}\left(\theta_{1} * \theta_{2}\right)\right]=\frac{1}{2 \pi i} \int_{-\infty}^{\infty} \hat{\theta}_{1}(\sigma) \hat{\theta}_{2}(\sigma) \partial_{\sigma} \log \varphi^{0}(i \sigma) d \sigma .
$$

Proof. This result is an extension of a mini-trace formula proved in Theorem 9.11 of [LP1] (see also [LP2]) for $C^{(\infty)}$ functions $\psi$ with compact support in $(0, \infty)$,

$$
\operatorname{tr}\left[Z^{0}(\psi)\right]=\frac{1}{2 \pi i} \int_{-\infty}^{\infty} \hat{\psi}(\sigma) \partial_{\sigma} \log \varphi^{0}(i \sigma) d \sigma
$$

It is sufficient therefore to obtain (4.19) in the limit from $(4.19)^{\prime}$. Choose $\chi_{n}(t)$ to be smooth, equal to 1 for $t \leq n$ and 0 for $t \geq n+1$, and define $\theta_{n}^{\prime}=\chi_{n} \theta_{1}$, $\theta_{n}^{\prime \prime}=\chi_{n} \theta_{2}$. Then

$$
\left|\theta_{n}^{\prime}-\theta_{1}\right|_{2} \rightarrow 0 \text { and }\left|\theta_{n}^{\prime \prime}-\theta_{2}\right|_{2} \rightarrow 0
$$

and therefore, according to Lemma 4.3,

$$
\left\|Z\left(\theta_{n}^{\prime}\right)-Z\left(\theta_{1}\right)\right\|_{2} \rightarrow 0 \text { and }\left\|Z\left(\theta_{n}^{\prime \prime}\right)-Z\left(\theta_{2}\right)\right\|_{2} \rightarrow 0 .
$$

It follows that

$$
\operatorname{tr}\left[Z\left(\theta_{n}^{\prime} * \theta_{n}^{\prime \prime}\right)\right] \rightarrow \operatorname{tr}\left[Z\left(\theta_{1} * \theta_{2}\right)\right]
$$

As for the right-hand side in (4.19), it is known from the Weyl asymptotic formula that the growth of the winding number of $\varphi^{0}$ is $O\left(|\sigma|^{2}\right)$, see [LP1]. Since $\left|\hat{\theta}_{n}^{\prime}(\sigma) \hat{\theta}_{n}^{\prime \prime}(\sigma)\right| \leq \mathrm{const} /\left(1+|\sigma|^{4}\right)$ uniformly in $n$, we see that the right-hand side also converges.

Next we approximate $\theta_{n}^{\prime} * \theta_{n}^{\prime \prime}$ by $\theta_{n}^{\prime} * \theta_{n}^{\prime \prime} * \psi_{\varepsilon}$, where $\psi_{\varepsilon}$ is a $C^{(\infty)}$ approximation of the identity with compact support in $(0, \infty)$. Since $Z\left(\psi_{\varepsilon}\right)$ approximates $I$ strongly, we can assert that

$$
\operatorname{tr}\left[Z\left(\theta_{n}^{\prime} * \theta_{n}^{\prime \prime} * \psi_{\varepsilon}\right)\right] \rightarrow \operatorname{tr}\left[Z\left(\theta_{n}^{\prime} * \theta_{n}^{\prime \prime}\right)\right]
$$

Again by bounded convergence it is clear that the right hand side in (4.19) also converges. This completes the proof of Theorem 4.6. 
According to a theorem of Lidskii $[\mathrm{GK}]$ the trace of an operator of trace class is equal to the sum of its eigenvalues, each being counted with multiplicity equal to the dimension of its eigenspace. If $\lambda$ is an eigenvalue of $B^{0}$ then $e^{\lambda t}$ is an eigenvalue of $Z^{0}(t)$ and $\hat{\psi}(-i \lambda)$ is the corresponding eigenvalue of $Z^{0}(\psi)$.

The eigenvalues of $Z_{+}$and $Z_{0}$ have been characterized in Chapter 3 of [LP1]. According to Lemma 3.18 the eigenvalues of $B_{+}$are simple and are equal to the $-\lambda_{i}$ 's of (4.6) corresponding to the noncuspidal solutions $q_{j}$ (the so-called relevant eigenvalues). According to Lemmas 3.16 and 3.17 of [LP1] the eigenspaces of $B_{0}$ are in one-to-one correspondence with the $\operatorname{Re} \lambda<0$ eigenspaces of $B$. It should be pointed out that Lemma 3.16 is stated in terms of a dual operator $B_{-}^{0}$, which has the same eigenvalues and multiplicities as $B_{0}$. In fact, $B_{0}$ is the adjoint of $R B_{-}^{0} R$ since

$$
R Z_{-}^{0}(t) R=R P_{+}^{\prime} U(t) P_{-}^{\prime \prime} R=P_{-}^{\prime} U(-t) P_{+}^{\prime \prime}=\left(Z_{0}(t)\right)^{*} .
$$

As a corollary to Theorem 4.6 we therefore have

Corollary 4.7. For $\psi=\theta_{1} * \theta_{2}, \theta_{1}, \theta_{2}$ in $C_{0}^{(2)}[0, \infty)$,

$$
\sum \hat{\psi}\left(-i \mu_{j}\right)=\frac{1}{2 \pi i} \int \hat{\psi}(\sigma) \partial_{\sigma} \log \varphi_{0}(i \sigma) d \sigma,
$$

where the sum is over the $\operatorname{Re} \mu<0$ eigenvalues of $B_{0}$, and

$$
\sum \hat{\psi}\left(-i \lambda_{j}\right)=\frac{1}{2 \pi i} \int \hat{\psi}(\sigma) \partial_{\sigma} \log \varphi_{+}(i \sigma) d \sigma,
$$

where the sum is over the relevant $\lambda_{j}$ 's.

Proof of Theorem 4.1. Again let $Z^{0}$ denote either $Z_{0}$ or $Z_{+}$and set $\psi(t)=$ $e^{-\lambda t}$ for $t \geq 0$ and $\psi(t)=0$ for $t<0$. For $\operatorname{Re} \lambda>0$

$$
Z^{0}(\psi)=R_{\lambda}\left(B^{0}\right) \text {. }
$$

Then $\theta=\psi * \psi$ belongs to $C_{0}^{(2)}[0, \infty)$ and $\hat{\theta}(\sigma)=(\lambda-i \sigma)^{-2}$.

We now apply the mini-trace formula to $Z^{0}(\theta * \theta)=R_{\lambda}^{4}\left(B^{0}\right)$,

$$
\operatorname{tr}\left[R_{\lambda}^{4}\left(B^{0}\right)\right]=\frac{1}{2 \pi i} \int(\lambda-i \sigma)^{-4} \partial_{\sigma} \log \varphi^{0}(i \sigma) d \sigma .
$$

The scattering matrix $\Phi^{0}(\sigma)$ is meromorphic with poles at the spectral points of $B^{0}$. We can therefore change the path of integration by pushing a small tube $T_{\mu}$ into the left halfplane, which contains no spectral points of $B^{0}$ except $\mu$. Then (4.22) becomes

$$
\operatorname{tr}\left[R_{\lambda}^{4}\left(B^{0}\right)\right]=\frac{1}{2 \pi i} \int(\lambda-i \sigma)^{-4} \partial_{\sigma} \log \varphi^{0}(i \sigma) d \sigma+\operatorname{Or}\left(\varphi^{0}, \mu\right)(\lambda-\mu)^{-4}
$$

here $\operatorname{Or}\left(\varphi^{0}, \mu\right)$ denotes the order of the pole of $\varphi^{0}$ at $\mu$ (or the negative of the order of the zero). The first term on the right is analytic for $\lambda$ in the 
right halfplane and in $T_{\mu}$. The resolvent is meromorphic having poles at the spectrum of $B^{0}$ of the type

$$
\begin{aligned}
R_{\lambda}\left(B^{0}\right)=P_{\mu} /(\lambda-\mu) & + \text { higher powers of }(\lambda-\mu)^{-1} \\
& + \text { regular term } ;
\end{aligned}
$$

$P_{\mu}$ is a projection onto the eigenspace of $B^{0}$, acting like the identity on the second term in (4.24) and annihilating the third term. Clearly

$$
\operatorname{tr}\left[P_{\mu}\right]=m^{0}(\mu) \text {. }
$$

Using the fact that

$$
|\operatorname{tr}[C D]| \leq\|C\|_{1}\|D\|,
$$

for $C$ of trace class and $D(\lambda)$ continuous in the operator norm, it is easy to show that

$$
\int \operatorname{tr}[C D(\lambda)] d \lambda=\operatorname{tr}\left[\int C D(\lambda) d \lambda\right] .
$$

We apply this to the integral of

$$
R_{\lambda}^{4}\left(B^{0}\right)=R_{\lambda_{0}}^{4}\left(B^{0}\right)\left(I+\left(\lambda-\lambda_{0}\right) R_{\lambda_{0}}\left(B^{0}\right)\right)^{-4}
$$

over a small circle $C_{\mu}$ about $\mu$, contained in $T_{\mu}$. Multiplying (4.23) through by $(\lambda-\mu)^{3} / 2 \pi i$ and integrating over $C_{\mu}$ we obtain

$$
m^{0}(\mu)=\operatorname{Or}\left(\varphi^{0}, \mu\right) \text {. }
$$

The remainder of the proof of Theorem 4.1 is a matter of bookkeeping. We apply (4.28) to both $B_{0}$ and $B_{+}$. The spectral representation of (4.16) shows that $\Phi=\Phi_{+}^{-1} \Phi_{0}$ and hence that

$$
\varphi(\lambda)=\varphi_{0}(\lambda) / \varphi_{+}(\lambda) .
$$

It follows that

$$
\operatorname{Or}(\varphi, \mu)=\operatorname{Or}\left(\varphi_{0}, \mu\right)-\operatorname{Or}\left(\varphi_{+}, \mu\right)
$$

Finally recall that $\Phi^{0}$ is an inner function. This implies that $\varphi^{0}(\lambda) \varphi^{0}(-\bar{\lambda})=1$ and hence that $\operatorname{Or}\left(\varphi^{0}, \lambda\right)=-\operatorname{Or}\left(\varphi^{0},-\bar{\lambda}\right)$.

A point $\mu$ is a spectral point of $B$ in the left halfplane, not on $\left[-\frac{1}{2}, 0\right)$, iff it belongs to the spectrum of $B_{0}$ (it is not in the spectrum $B_{+}$); the corresponding eigenspaces have the same multiplicity. For such points

$$
m(\mu)=m_{0}(\mu)=\operatorname{Or}\left(\phi_{0}, \mu\right)=\operatorname{Or}\left(\phi^{\prime}, \mu\right) .
$$

A point $\mu$ in $\left[-\frac{1}{2}, 0\right)$ belongs to the spectrum of $B$ if it is a scattering frequency (and hence in the spectrum of $B_{0}$ ) or a cusp form eigendata. If it is a cusp form eigendata it will also correspond through the time reversal operator $R$ to a cusp form eigendata at $-\mu$ and will contribute to the multiplicity of $-\mu$. In taking the difference $m(\mu)-m(-\mu)$ the contributions of the cusp form 
eigendata cancel out. It may also happen that $\mu=-\lambda_{j}$ for some relevant $\lambda_{j}$. In this case $\mu$ will be a pole of $\phi_{+}$. Summing up we see that

$$
\begin{aligned}
m(\mu)-m(-\mu) & =m_{0}(\mu)-m_{+}(\mu)=\operatorname{Or}\left(\phi_{0}, \mu\right)-\operatorname{Or}\left(\phi_{+}, \mu\right) \\
& =\operatorname{Or}(\phi, \mu)=-\operatorname{Or}(\phi,-\mu) .
\end{aligned}
$$

This concludes the proof of Theorem 4.1.

Proof of Lemma 4.3. For $\theta$ in $C_{0}^{(2)}[0, \infty)$ we have

$$
\begin{aligned}
B Z(\theta) & =\lim \frac{Z(\delta)-I}{\delta} Z(\theta)=-\int_{0}^{\infty} \theta^{\prime}(t) Z(t) d t, \\
B^{2} Z(\theta) & =\int_{0}^{\infty} \theta^{\prime \prime}(t) Z(t) d t+\theta^{\prime}(0) I,
\end{aligned}
$$

and it follows from (2.21) and (4.1) that

$$
\|B Z(\theta)\|_{G} \leq|\theta|_{2} \text { and }\left\|B^{2} Z(\theta)\right\|_{G} \leq 2|\theta|_{2}
$$

in the $G$-norm.

Choose $M=F \backslash[$ cusps above $y=a]$ and set $\left\{u_{0}, v_{0}\right\}=Z(\theta) f$. Since

$$
u_{0}^{(0)}(y)=c y^{1 / 2}, \quad v_{0}^{(0)}(y)=0 \text { for } y>a
$$

in each cusp, we see from (2.7) and (2.8) that this part of $\left\{u_{0}, v_{0}\right\}$ does not contribute to its $G$-norm. Hence if we denote $\left\{u_{0}, v_{0}\right\}$ minus the zeroth Fourier coefficient (4.35) by $\{u, v\}$ and simply assume that the domain for the zero Fourier coefficients ends at $y=a$ in each cusp, we will not effect the $G$-norm estimates.

We shall, however, need some interior elliptic estimates that include the values of $u^{(0)}$ up to $y=a$ in the cusps. To get around the obvious fact that $[z ; y=a]$ is no longer interior to the domain we introduce a new set of incoming and outgoing subspaces, defined as in (2.10) but with $a$ replaced by $b>a$. Let $P_{b}$ denote the analogue of $P_{a}=P$ and set $Z_{b}(t)=P_{b} U(t) P_{b}$, etc. Notice that $P_{a} P_{b}=P_{b} P_{a}=P_{a}$, and hence that $Z_{a}(t)=P_{a} Z_{b}(t) P_{a}$ and $K_{b} \supset K_{a}$. Moreover $\left\{u_{0}, v_{0}\right\}=P_{a} Z_{b}(\theta) f$ for $f$ in $K_{a}$. Now the region $[y<a]$ is interior to $[y<b]$, and hence the elliptic estimates on $\{u, v\}$ can be thought of as interior estimates taken from $Z_{b}(\theta) f$.

In our notation $B^{2} Z(\theta) f=\{L u, L v\}$ and by (4.34)

$$
\|\{u, v\}\|_{G},\|\{L u, L v\}\|_{G} \leq c\|f\|_{G} .
$$

It therefore follows by elliptic theory that

$$
\sum_{|\alpha| \leq 3} \int_{M}\left|\partial^{\alpha} u\right|^{2} d x d y, \sum_{|\alpha| \leq 2} \int_{M}\left|\partial^{\alpha} v\right|^{2} d x d y \leq c^{\prime}\|f\|_{G}^{2} .
$$

Next choose $\xi(y)$ in $C^{(\infty)}$ so that

$$
\xi(y)= \begin{cases}1 & \text { for } y \geq a \\ 0 & \text { for } y \leq a-1\end{cases}
$$


and set $\left\{u_{1}, v_{1}\right\}=\{\xi u, \xi v\}$-zero Fourier component, in each cusp. It is clear from (4.37) that inequalities analogous to (4.36) hold for $\left\{u_{1}, v_{1}\right\}$. Expanding $\left\{u_{1}, v_{1}\right\}$ into a Fourier series in the $x$-variable, we see from (2.7) that

$$
\int\left|u_{1}\right|^{2} d x d y \leq \sum_{k} \int\left|u_{1}^{(k)}\right|^{2} d y \leq c^{\prime}\|f\|_{G}^{2} .
$$

Applying this inequality to $L u_{1}$, we obtain

$$
\int y^{4}\left|\Delta_{0} u_{1}\right|^{2} d x d y \leq 2 \int\left(\left|L u_{1}\right|^{2}+\left|u_{1}\right|^{2}\right) d x d y \leq c\|f\|_{G}^{2} .
$$

Next set $y^{2} \Delta_{0} u_{1}=g \in L^{2}$, multiply through by $u_{1}$, and integrate by parts,

$$
\begin{aligned}
\int y^{2} u_{1} \partial_{y}^{2} u_{1} d y & =-\int y^{2}\left|\partial_{y} u_{1}\right|^{2} d y-2 \int y u_{1} \partial_{y} u_{1} d y \\
& =-\int y^{2}\left|\partial_{y} u_{1}\right|^{2} d y+\int\left|u_{1}\right|^{2} d y
\end{aligned}
$$

This together with a similar calculation for $\int y^{2} u_{1} \partial_{x}^{2} u_{1} d x$ gives

$$
\begin{aligned}
\int y^{2}\left(\left|\partial_{y} u_{1}\right|^{2}+\left|\partial_{x} u_{1}\right|^{2}\right) d x d y & \leq \int\left(|g|^{2}+2\left|u_{1}\right|^{2}\right) d x d y \\
& \leq c\|f\|_{G}^{2} .
\end{aligned}
$$

A calculation analogous to that of (4.39) now yields

$$
\int y^{2}\left|u_{1}\right|^{2} d x d y \leq c\|f\|_{G}^{2}
$$

We need one last calculation. Combining

$$
\begin{aligned}
y^{4}\left|\Delta_{0}\left(\partial_{y} u_{1}\right)\right|^{2} & \leq 2 y\left|\partial_{y}\left(\frac{y^{2} \Delta_{0} u_{1}}{y^{1 / 2}}\right)\right|^{2}+5 y^{2}\left|\Delta_{0} u_{1}\right|^{2} \\
& \leq 4 y\left|\partial_{y}\left(\frac{L u_{1}}{y^{1 / 2}}\right)\right|^{2}+y\left|\partial_{y}\left(\frac{u_{1}}{y^{1 / 2}}\right)\right|^{2}+5 y^{2}\left|\Delta_{0} u_{1}\right|^{2}
\end{aligned}
$$

and the $x$ derivative analogue with (4.40) we get

$$
\begin{aligned}
& \int y^{4}\left(\left|\Delta_{0}\left(\partial_{y} u_{1}\right)\right|^{2}+\left|\Delta_{0}\left(\partial_{x} u_{1}\right)\right|^{2}\right) d x d y \\
& \quad \leq 4 E\left(\left\{L u_{1}, 0\right\}\right)+\int\left(y\left|\partial_{y}\left(\frac{u_{1}}{y^{1 / 2}}\right)\right|^{2}+5 y^{2}\left|\Delta_{0} u_{1}\right|^{2}\right) d x d y \\
& \leq c\|f\|_{G}^{2} .
\end{aligned}
$$

Applying the Sobolev lemma to (4.40), (4.42), and (4.43) we finally obtain

$$
\left|u_{1}(z)\right| \leq(c / y)\|f\|_{G} \text { and }\left|\partial u_{1}(z)\right| \leq(c / y)\|f\|_{G}
$$

in each cusp. For the same reason the inequalities (4.37) imply

$$
|u(z)| \leq c\|f\|_{G}
$$

$$
|\partial u(z)| \leq c\|f\|_{G}
$$

$$
|v(z)| \leq c\|f\|_{G}
$$

in $M$. 
As for $v_{1}$ in the cusps, we know that $v_{1}$ is the first component of $B Z(\theta)$ and hence, as in (4.39),

$$
\int\left|v_{1}\right|^{2} d x d y \leq c\|f\|_{G}
$$

In addition $L v_{1}=y^{2} \Delta_{0} v_{1}+\frac{1}{4} v_{1}$ lies in $L^{2}\left(d x d y / y^{2}\right)$. From this we see that

$$
\int y^{2}\left|\Delta_{0} v_{1}\right|^{2} d x d y \leq c\|f\|_{G}
$$

Again applying the Sobolev lemma, this time to (4.46) and (4.47), we get

$$
\left|v_{1}(z)\right| \leq c\|f\|_{G}
$$

in the cusps.

The inequalities (4.44), (4.45), and (4.48) prove that $u(z)$ and $v(z)$ are bounded linear functionals of $\left\{f_{1}, f_{2}\right\}$,

$$
\begin{aligned}
& u_{1}(z)=G\left(\left\{k_{11}(z, \cdot), k_{12}(z, \cdot)\right\},\left\{f_{1}, f_{2}\right\}\right), \\
& v_{1}(z)=G\left(\left\{k_{21}(z, \cdot), k_{22}(z, \cdot)\right\},\left\{f_{1}, f_{2}\right\}\right),
\end{aligned}
$$

with norms

$$
\begin{gathered}
\left\|\left\{k_{11}(z, \cdot), k_{12}(z, \cdot)\right\}\right\|_{G},\left\|\left\{\partial_{z} k_{11}(z, \cdot), \partial_{z} k_{12}(z, \cdot)\right\}\right\|_{G} \leq c / y, \\
\left\|\left\{k_{21}(z, \cdot), k_{22}(z, \cdot)\right\}\right\|_{G} \leq c .
\end{gathered}
$$

Let $\left[\Psi_{j}\right]$ be a complete orthonormal basis for $H_{G}$. Then the Hilbert Schmidt norm of $Z(\theta)$ is defined as

$$
\|Z(\theta)\|_{2}^{2}=\sum_{j} G_{z}\left(\left\{G\left(\left\{k_{11}(z, \cdot), k_{12}(z, \cdot)\right\}, \Psi_{j}\right), G\left(\left\{k_{21}(z, \cdot), k_{22}(z, \cdot)\right\}, \Psi_{j}\right)\right\}\right),
$$

which consists of terms like

$$
\begin{aligned}
\int\left(\sum_{j} y\left|G\left(\left\{\partial_{z}\left(\frac{k_{11}(z, \cdot)}{y^{1 / 2}}\right), \partial_{z}\left(\frac{k_{12}(z, \cdot)}{y^{1 / 2}}\right)\right\}, \Psi_{j}\right)\right|^{2}\right. \\
\left.\quad+\sum_{j}\left|G\left(\left\{k_{21}(z, \cdot), k_{22}(z, \cdot)\right\}, \Psi_{j}\right)\right|^{2} / y^{2}\right) d x d y .
\end{aligned}
$$

It follows from (4.50) via the Parseval relation that these terms are finite and hence that $Z(\theta)$ is Hilbert Schmidt.

Remark. In Theorem 9.10 of [LP1] a trace formula is derived for the operator $B$. On the left-hand side of this formula are the various values of $h(-i \lambda)$ at the corresponding spectral points of $B$ weighted according to their multiplicities. In particular $h(0)$ occurs with the multiplicity of the null space (see [LP1, equation (9.118)]). By comparing this with (1.1) and making use of Corollaries 3.4 and 4.2 (for $r \neq 0$ ) one can obtain an alternate proof of the fact, stated in Theorem 3.2, that $m(0)=p+2 q$.

\section{Perturbation theory}

In the previous sections we have shown that the singular set $i \sigma(\Gamma)$ is the same as the spectrum of $B$. The real analyticity of $\sigma(\Gamma)$ will therefore follow 
by standard perturbation theory from the real analyticity of the resolvent of $B(\Gamma)$.

Ahlfors has developed a method for creating a quasi-conformal analytic deformation $\Gamma_{\tau}$ in Teichmüller space $T\left(\Gamma_{0}\right)$, generated by any one of the canonical elements of the tangent space at $\Gamma_{0}$ in $T\left(\Gamma_{0}\right)$. The corresponding metrics $g(\tau)$ on $F=\Gamma_{0} \backslash H$ vary real analytically in $\tau$. Because of the simple structure of a cusp, it is possible (see [PS1, Lemma 2.2]) to construct an analytic set of diffeomorphisms $\varphi_{\tau}$ in $F$ such that in each cusp $\hat{g}(\tau)=\varphi_{\tau}^{*}(g(\tau))$ is equal to $g(0)$ for $y>a$ and to $g(\tau)$ for $y<a / 2$. The Laplace-Beltrami operators $L_{0}(\tau)$ corresponding to $\hat{g}(\tau)$ are second-order differential operators with real analytic coefficients, which are equal to the standard $y^{2} \Delta_{0}$ for $y>a$ in the cusps. We set

$$
L(\tau)=L_{0}(\tau)+\frac{1}{4}
$$

As defined in (2.3), $E$ and hence $G$ depend on $L(\tau)$. However, since $L(\tau)=y^{2} \Delta_{0}+\frac{1}{4}$ in the cusps, the $E_{j}, j \neq 0$, as given in (2.7), do not depend on $\tau$. Since the $\hat{g}(\tau)$ are quasi-isometric on $M$, the $G_{\tau}$ 's will define equivalent norms on $F$. In what follows we shall fix $E$ and $G$ as defined by $L(0)$ and work with the resulting fixed Hilbert space $H_{G}$.

The generators for the solution operators of the wave equation are now

$$
A_{\tau}=\left(\begin{array}{cc}
0 & I \\
L(\tau) & 0
\end{array}\right)
$$

Notice that $A_{0}$ is skew symmetric with respect to $E$, whereas for $\tau \neq 0$, $A_{\tau}$ need not be. Since $L(\tau)=y^{2} \Delta_{0}+\frac{1}{4}$ for $y>a$ in each cusp, we can define incoming and outgoing subspaces as in (2.10) - they do not depend on $\tau$. Defining $P$ as in (2.19) we set

$$
Z_{\tau}(t)=P U_{\tau}(t) P
$$

and denote its generator by $B_{\tau}$.

Theorem 5.1. $R_{\lambda}\left(B_{\tau}\right)$ is real analytic in $\tau$ on the resolvent set of $B_{0}$ for $|\tau|$ sufficiently small.

Proof. Let $\lambda$ belong to the resolvent set of $B_{0}$ and set $\{u, v\}=R_{\lambda}\left(B_{0}\right) f$. Then

$$
\lambda^{2} u-L(0) u=\lambda f_{1}+f_{2},
$$

and we conclude by elliptic theory as in (4.37) that

$$
\sum_{|\alpha| \leq 2} \int_{M}\left|\partial^{\alpha} u\right|^{2} d x d y \leq c\|f\|_{G}^{2}
$$

Notice that

$$
B_{\tau}-B_{0}= \begin{cases}0 & \text { for } y>a \text { in the cusps } \\
\left(\begin{array}{ccc}
0 & 0 \\
L(\tau)-L(0) & 0
\end{array}\right) & \text { elsewhere. }\end{cases}
$$


It follows that $B_{\tau} R_{\lambda}\left(B_{0}\right)$ is real analytic in the strong and hence in the uniform operator topology, and it follows from this that

$$
R_{\lambda}\left(B_{\tau}\right)=R_{\lambda}\left(B_{0}\right)\left(I-\left(B_{\tau}-B_{0}\right) R_{\lambda}\left(B_{0}\right)\right)^{-1}
$$

and is real analytic in $\tau$.

Actually $R_{\lambda}\left(B_{\tau}\right)$ is analytic jointly in both $\lambda$ and $\tau$. In fact, for $\lambda_{0}$ in the resolvent set of $B_{0}$, Theorem 5.1 implies that $R_{\lambda_{0}}\left(B_{\tau}\right)$ will be bounded for $|\tau| \leq \delta$, and since the expression

$$
R_{\lambda}\left(B_{\tau}\right)=R_{\lambda_{0}}\left(B_{\tau}\right) \sum_{k}\left(\lambda-\lambda_{0}\right)^{k} R_{\lambda_{0}}^{k}\left(B_{\tau}\right)
$$

converges absolutely for $\left|\lambda-\lambda_{0}\right| \leq \varepsilon$, the joint analyticity follows.

For $\lambda_{0}$ in the spectrum of $B_{0}$, let $C_{0}$ denote a small circle about $\lambda_{0}$ that contains no other point in the spectrum of $B_{0}$. Then

$$
Q_{\tau}=\frac{1}{2 \pi i} \int_{C_{0}} R_{\lambda}\left(B_{\tau}\right) d \lambda
$$

projects onto the span of the eigenspaces of $B_{\tau}$ with eigenvalues in $C_{0}$ and $Q_{\tau} B_{\tau}$ is real analytic and is equal to $B_{\tau}$ on these eigenspaces and to 0 on the range of $\left(I-Q_{\tau}\right)$. According to Theorem 1.8 of Chapter 7 in Kato [K], an immediate consequence of this is

Corollary 5.2. If $\lambda(0)$ is an eigenvalue of $B_{0}$ of multiplicity one, then $\lambda(\tau)$ and the associated eigenfunction $f_{\tau}$ are real analytic in $\tau$ for $|\tau|$ sufficiently small. If the multiplicity of $\lambda(0)$ is greater than one, then the eigenvalues decompose into a finite system of real analytic functions having at most algebraic singularities.

The remainder of this section is devoted to a proof of Fermi's Golden Rule, which provides us with an explicit expression for

$$
\left.\operatorname{Re} \frac{d^{2}}{d \tau^{2}} \lambda(\tau)\right|_{\tau=0},
$$

when $\lambda(0)=i \sigma$ is a purely imaginary nonzero eigenvalue of $B_{0}$ of multiplicity one. As we have shown in $\S 3, \operatorname{Re} \lambda \leq 0$ for $\lambda$ near $i \sigma$ so that $\dot{\lambda}(\tau)$ is purely imaginary at $\tau=0$. Thus the expression (5.9) gives the rate at which $\lambda(\tau)$ leaves the imaginary axis to become a resonance. Our proof is modelled after that of Simon (see [RS]).

In this case $\lambda(\tau)$ and the associated normalized eigenfunction $f_{\tau}$ are real analytic,

$$
\left(B_{\tau}-\lambda(\tau)\right) f_{\tau}=0
$$

Differentiating we obtain

$$
\left(B_{\tau}-\lambda(\tau)\right) \dot{f}_{\tau}+(\dot{B}-\dot{\lambda}(\tau)) f_{\tau}=0
$$

Recall from (2.27) that $E$-adjoint $B^{*}=R B R$. Since $f_{0}$ and $R f_{0}$ are cusp forms,

$$
\begin{aligned}
& B_{0} f_{0}=A_{0} f_{0}=i \sigma f_{0} \\
& B_{0}^{*} f_{0}=R B_{0} R f_{0}=R A_{0} R f_{0}=-i \sigma f_{0}
\end{aligned}
$$


Hence setting $\tau=0$ in (5.11) and taking the $E$-inner product with $f_{0}$, we get

$$
\dot{\lambda}(0)=E\left(\dot{B}_{0} f_{0}, f_{0}\right) .
$$

This shows that $\left(\dot{B}_{0}-\dot{\lambda}(0)\right) f_{0}$ is $E$-orthogonal to $f_{0}$ so that we can invert $(5.11)$ (at $\tau=0$ ) to obtain

$$
\dot{f}_{0}=R_{i \sigma}^{0}\left(B_{0}\right)\left(\dot{B}_{0}-\dot{\lambda}(0)\right) f_{0}
$$

where

$$
R_{\lambda}^{0}\left(B_{0}\right)=\left(I-Q_{0}\right) R_{\lambda}\left(B_{0}\right)=R_{\lambda}\left(B_{0}\right)-Q_{0} /(\lambda-i \sigma) .
$$

Finally we take the second derivative of (5.10) with respect to $\tau$,

$$
\left(B_{0}-\lambda(0)\right) \ddot{f}_{0}+2\left(\dot{B}_{0}-\dot{\lambda}(0)\right) \dot{f}_{0}+\left(\ddot{B}_{0}-\ddot{\lambda}(0)\right) f_{0}=0 \text {. }
$$

Taking the $E$-inner product with respect to $f_{0}$, we get an expression for $\ddot{\lambda}(0)$,

$$
\ddot{\lambda}(0)=E\left(\ddot{B}_{0} f_{0}, f_{0}\right)+2 E\left(\left(\dot{B}_{0}-\dot{\lambda}(0)\right) R_{i \sigma}^{0}\left(B_{0}\right)\left(\dot{B}_{0}-\dot{\lambda}(0)\right) f_{0}, f_{0}\right) .
$$

We are interested in the real part of $\ddot{\lambda}(0)$, which is considerably simpler than (5.17). In the first place $f_{0,2}=i \sigma f_{0,1}$ so that

$$
E\left(\ddot{B}_{0} f_{0}, f_{0}\right)=\left(\ddot{L}(0) f_{0,1}, f_{0,2}\right)=-i \sigma\left(\ddot{L}(0) f_{0,1}, f_{0,1}\right),
$$

which is purely imaginary. Moreover since $f_{0}$ is a cusp form,

$$
\dot{B}_{0}^{*} f_{0}=R \dot{B}_{0} R f_{0}=R \dot{A}_{0} R f_{0}=\left\{0,-\dot{L}(0) f_{0,1}\right\}=-\dot{B}_{0} f_{0} \text {. }
$$

Hence

$$
\operatorname{Re} \ddot{\lambda}(0)=2 \operatorname{Re} E\left(R_{i \sigma}^{0}\left(B_{0}\right)\left(\dot{\lambda}(0)-\dot{B}_{0}\right) f_{0},\left(\overline{\dot{\lambda}(0)}+\dot{B}_{0}\right) f_{0}\right) .
$$

Next we show that $Q_{0}$ is an $E$-orthogonal projection. If we denote by $H_{p}$ the closed linear span of the cusp form eigenfunctions of $B_{0}$, then $R_{\lambda}\left(B_{0}\right) H_{p} \subset H_{p}$ and, since $R H_{p}=H_{p}, R_{\lambda}^{*}\left(B_{0}\right) H_{p}=R R_{\bar{\lambda}}\left(-B_{0}\right) R H_{p} \subset H_{p}$. It follows that $R_{\lambda}\left(B_{0}\right)$ takes the $E$-orthogonal complement of $H_{p}$ into itself and so does $Q_{0}$. Since the range of $Q_{0}$ is in $H_{p}, Q_{0}$ must annihilate $H_{p}^{\perp}$. Now $B_{0}$ and $A_{0}$ are the same on $H_{p}$ so that

$$
\left.Q_{0}\right|_{H_{p}}=\left.\frac{1}{2 \pi i} \int_{C_{0}} R_{\lambda}\left(A_{0}\right)\right|_{H_{p}} d \lambda,
$$

which is $E$-orthogonal on $H_{p}$. We conclude that $Q_{0}$ is an $E$-orthogonal projection on all of $H_{G}$. Thus both $R_{\lambda}^{0}\left(B_{0}\right)$ and its adjoint annihilate $f_{0}$ and (5.20) can now be rewritten as

$$
\operatorname{Re} \ddot{\lambda}(0)=-2 \operatorname{Re} E\left(R_{i \sigma}^{0}\left(B_{0}\right) \dot{B}_{0} f_{0}, \dot{B}_{0} f_{0}\right) .
$$

According to (2.22), $R_{\lambda}\left(B_{0}\right)=P R_{\lambda}\left(A_{0}\right) P$ for $\operatorname{Re} \lambda>\omega$ and by analytic continuation it continues to hold for $\operatorname{Re} \lambda>0$. Further it is clear that $P Q_{0}=$ $Q_{0} P=Q_{0}$ and that $P \dot{B}_{0}=\dot{B}_{0} P=\dot{B}_{0}$. Hence setting

$$
R_{\lambda}^{0}\left(A_{0}\right)=R_{\lambda}\left(A_{0}\right)-Q_{0} /(\lambda-i \sigma),
$$


we see that $(5.21)$ can be rewritten as

$$
\begin{aligned}
\operatorname{Re} \ddot{\lambda}(0) & =-2 \lim _{\varepsilon \searrow 0} \operatorname{Re} E\left(R_{i \sigma+\varepsilon}^{0}\left(B_{0}\right) \dot{B}_{0} f_{0}, \dot{B}_{0} f_{0}\right) \\
& =-2 \lim _{\varepsilon \searrow 0} \operatorname{Re} E\left(R_{i \sigma+\varepsilon}^{0}\left(A_{0}\right) \dot{B}_{0} f_{0}, \dot{B}_{0} f_{0}\right) \\
& =-\lim _{\varepsilon \searrow 0}\left[E\left(R_{i \sigma+\varepsilon}^{0}\left(A_{0}\right) \dot{B}_{0} f_{0}, \dot{B}_{0} f_{0}\right)-E\left(R_{i \sigma-\varepsilon}^{0}\left(A_{0}\right) \dot{B}_{0} f_{0}, \dot{B}_{0} f_{0}\right)\right]
\end{aligned}
$$

since

$$
R_{i \sigma+\varepsilon}^{0 *}\left(A_{0}\right)=R_{-i \sigma+\varepsilon}^{0}\left(-A_{0}\right)=-R_{i \sigma-\varepsilon}^{0}\left(A_{0}\right) .
$$

Now the contributions to (5.23) of the point spectra of $A_{0}$ (including $i \sigma$ ) vanish in the limit. We can therefore replace $\dot{B}_{0} f_{0}$ by $Q^{\prime} B_{0} f_{0}$, where as in $\S 4$, $Q^{\prime}$ projects $H_{G}$ onto $H_{c}^{\prime}$. Recall that $A_{0}$ is skew-selfadjoint on $H_{c}^{\prime}$ with a spectral representation given in terms of the Eisenstein series $e_{0}^{k}(z)$ (see [LP1, Theorem 8.1]): For $g$ in $H_{G}$

$$
\left(\widetilde{Q^{\prime} g}\right)^{k}(s)=E\left(g, e^{k}(s)\right)
$$

where

$$
e^{k}(s)=\frac{a^{-i s}}{2 \sqrt{\pi}}\left\{\frac{1}{i s}, 1\right\} e_{0}^{k}\left(\frac{1}{2}+i s\right) .
$$

In terms of the spectral representation, (5.22) becomes

$$
\begin{aligned}
\operatorname{Re} \ddot{\lambda}(0) & =-\lim _{\varepsilon \searrow 0} 2 \int_{-\infty}^{\infty} \frac{\varepsilon}{(s-\sigma)^{2}+\varepsilon^{2}} \sum_{k}\left|E\left(\dot{B}_{0} f_{0}, e^{k}(s)\right)\right|^{2} d s \\
& =-2 \pi \sum_{k}\left|E\left(\dot{B}_{0} f_{0}, e^{k}(\sigma)\right)\right|^{2}
\end{aligned}
$$

Since the spectrum of $B$ is independent of the cut-off value $a$, so is $\ddot{\lambda}(0)$. This suggests that we pass to the limit as $a \rightarrow \infty$ on the right-hand side of (5.26). To this end let $L_{1}(\tau)$ denote the Laplace-Beltrami operator corresponding to $g(\tau)$. Then

$$
L_{1}(\tau)=L_{0}(\tau) \text { except for } y>a / 2 \text { in the cusps. }
$$

In Lemma 2.3 of [PS1] it is proved that

$$
\begin{aligned}
\left|E\left(\dot{B}_{0}(a) f_{0}, e^{k}(\sigma)\right)\right| & =\frac{1}{2 \sqrt{\pi}}\left|\left(\dot{L}_{0}(0, a) f_{0,1}, e_{0}^{k}\left(\frac{1}{2}+i \sigma\right)\right)\right| \\
& \rightarrow \frac{1}{2 \sqrt{\pi}}\left|\left(\dot{L}_{1}(0) f_{0,1}, e_{0}^{k}\left(\frac{1}{2}+i \sigma\right)\right)\right| .
\end{aligned}
$$

We therefore have

$$
\operatorname{Re} \ddot{\lambda}(0)=-\frac{1}{2} \sum_{k}\left|\left(\dot{L}_{1}(0) f_{0,1}, e_{0}^{k}\left(\frac{1}{2}+i \sigma\right)\right)\right|^{2} .
$$


This is Fermi's Golden Rule. It asserts that to second order a cusp form is destroyed if and only if the right side of (5.29) is different from zero. It was shown in [PS1] that the expression

$$
\left(\dot{L}_{1}(0) f_{0,1}, e_{0}^{k}\left(\frac{1}{2}+i \sigma\right)\right)
$$

is, to within a nonzero factor, equal to a special value of a Rankin-Selberg $L$-function.

\section{Applications}

In the previous sections we have established the analytic dependence of the singular set $\sigma(\Gamma)$ as a function on the deformation (or Teichmüller) space $T(\Gamma)$. One of the important related problems concerns the dissolution of cusp forms into poles of Eisenstein series under such deformations. The condition for this that emerges from Fermi's Golden Rule, developed in $\S 5$, is precisely the one obtained in [PS1] (by a somewhat different method), and we have nothing more to add to this problem.

However our theory also allows us to study the integer-valued function

$$
\nu(\Gamma)=\left(n+\operatorname{tr}\left[\Phi\left(\frac{1}{2}, \Gamma\right)\right]\right) / 2
$$

on $T(\Gamma)$. This function counts the number of +1 eigenvalues of $\Phi\left(\frac{1}{2}, \Gamma\right)$ and is equal to zero if and only if all of the eigenvalues are -1 . According to Theorem 3.2, $\nu(\Gamma)$ also counts the number of noncuspidal null vectors of $B(\Gamma)$ and hence

$$
0 \leq \nu(\Gamma) \leq m(0, \Gamma) .
$$

Further note that if $\Gamma_{1} \subset \Gamma_{2}$ (of finite index) then

$$
\sigma\left(\Gamma_{1}\right) \supset \sigma\left(\Gamma_{2}\right)
$$

Next we introduce the subsets

$$
V_{j}=[\Gamma \in T(\Gamma) ; m(0, \Gamma) \geq j]
$$

for $j=0,1,2, \ldots$. Note that $T=V_{0} \supset V_{1} \supset \cdots \supset V_{j} \supset \cdots$ and there is a subsequence

$$
T=V_{0} \supsetneqq V_{j_{1}} \supsetneqq V_{j_{2}} \supsetneqq \cdots
$$

These $V_{j_{i}}$ are the subvarieties on which $m(0, \Gamma)$ jumps in value.

Theorem 6.1. $m(0, \Gamma)$ is upper semicontinuous on $T(\Gamma)$. Moreover for each $j$, $V_{j}$ is a real analytic subvariety of $T(\Gamma)$ and $V_{j}$ is eventually empty.

Proof. The upper semicontinuity of $m(0, \Gamma)$ is a well-known property of the spectrum of a continuous family of operators under perturbation. That $V_{j}$ is eventually empty is the statement that $m(0, \Gamma)$ has a uniform upper bound over $T(\Gamma)$. Clearly $\nu(\Gamma) \leq n$ (the number of cusps) and it is well known (see Buser [B]) that there are less than $c(\Gamma)$ cusp forms with real eigenvalues (i.e., eigenvalues of the Laplacian in $\left.\left[0, \frac{1}{4}\right]\right)$; here $c(\Gamma)$ depends only on the 
signature of $\Gamma$ and hence only on $T(\Gamma)$. Combining this fact with Theorem 3.2 we see that

$$
m(0, \Gamma) \leq 2 c(\Gamma)+n .
$$

In order to prove the real analyticity of the $V_{j}$, we can work locally, making use of the analyticity and compactness of the resolvent $R_{\lambda}\left(B_{\tau}\right)$. A projection of the type (5.8), with $C_{0}$ centered about $\lambda=0$ and acting on $B_{\tau}$, will reduce the problem to that of a finite-dimensional operator having the same spectral properties as $B_{\tau}$ near the origin. For matrices the result is clear. For example

$$
V_{1}=\left[\tau ; \operatorname{det}\left(B_{\tau}\right)=0\right]
$$

etc.

We remark that while $m(0, \Gamma)$ is upper semicontinuous and jumps in value on real analytic sets, we do not claim that the same holds true for $\nu(\Gamma)$. However when $V_{1}$ is a proper subvariety, then we see from $(6.2)$ that $\nu(\Gamma)=0$ outside of this subvariety which is of codimension at least one. In this case $m(0, \Gamma)$ and $\nu(\Gamma)$ are generically zero on $T(\Gamma)$. Note that it suffices to show that $m(0, \Gamma)=0$ at only one point (and hence in a neighborhood of that point) in $T(\Gamma)$ in order to deduce that it is zero generically.

To find $\Gamma$ 's for which $m(0, \Gamma)=0$ we look at congruence subgroups of $\Gamma(1)=\operatorname{SL}(2, \mathbb{Z})$, these being essentially the only groups for which $\Phi(s, \Gamma)$ has been computed (see Efrat [E], Hejhal [He], and Huxley [Hu1]). We will also use the following result about

$$
\Gamma(N)=\left[\left(\begin{array}{ll}
a & b \\
c & d
\end{array}\right) \in \mathrm{SL}(2, \mathbb{Z}) ;\left(\begin{array}{ll}
a & b \\
c & d
\end{array}\right) \equiv I \bmod N\right]
$$

due to Huxley [Hu2]:

Assertion 6.2. If $\Gamma(N) \subset \Gamma \subset \mathrm{SL}(2, \mathbb{Z}), 1 \leq N \leq 17$, then $\Gamma \backslash H$ carries no cusp forms with exceptional eigenvalues, in particular no cusp forms with eigenvalue 0 .

Case (i). The modular group. For $\Gamma(1)=\operatorname{SL}(2, \mathbb{Z})$ there is only one cusp and $\Phi(s)$ has an explicit representation in terms of the zeta function. One knows that $\Phi\left(\frac{1}{2}\right)=-1$ and hence, by the above remarks and Assertion 6.2, we have

$$
m(0, \Gamma(1))=\nu(\Gamma(1))=0 .
$$

The modular group is rigid so that its deformation space is trivial, i.e., $T(\Gamma(1))$ $=\Gamma(1)$, and there is no more to be said.

Case (ii). $\Gamma=\Gamma(1)^{\prime}$. Here $\Gamma(1)^{\prime}=[\Gamma(1), \Gamma(1)]$ is the commutator subgroup of $\Gamma(1)$. It is well known (see Rankin $[\mathrm{Ra}]$ ) that $\left[\Gamma(1): \Gamma(1)^{\prime}\right]=6$. A set of coset representatives is

$$
T^{j} \Gamma(1)^{\prime}, \quad j=0,1, \ldots, 5, \text { where } T=\left(\begin{array}{ll}
1 & 1 \\
0 & 1
\end{array}\right) .
$$

Further $\Gamma(1)^{\prime} \backslash H$ has only one cusp; its parabolic generator is $\left(\begin{array}{ll}1 & 6 \\ 0 & 1\end{array}\right)$ and its genus is one. In fact $T\left(\Gamma(1)^{\prime}\right)$ is the deformation space of the one-punctured 
torus. It follows from (6.8) that the Eisenstein series for $\Gamma(1)^{\prime}$ is the same as that of $\Gamma(1)$ and so $\Phi\left(\frac{1}{2}, \Gamma(1)^{\prime}\right)=-1$. Also $\Gamma(1)^{\prime} \supset \Gamma(6)$ and so by Assertion 6.2

$$
m\left(0, \Gamma(1)^{\prime}\right)=0
$$

We conclude that $\Phi\left(\frac{1}{2}, \Gamma(1)^{\prime}\right)=-1$ generically on $T\left(\Gamma(1)^{\prime}\right)$ (the latter is of complex dimension one).

Case (iii). $\Gamma=\Gamma(4)$. The genus of $\Gamma(4) \backslash H$ is zero. It has six cusps and $T(\Gamma(4))$ is the deformation space of the six-times punctured sphere. The calculations for $\Phi\left(\frac{1}{2}, \Gamma(4)\right)$ show that $\nu(\Gamma(4))=0$. Again by Assertion 6.2 we conclude that

$$
m(0, \Gamma(4))=0
$$

Hence generally $\nu(\Gamma)=0$ for $\Gamma$ in $T(\Gamma(4))$, i.e., the six eigenvalues of $\Phi\left(\frac{1}{2}, \Gamma\right)$ are all -1 , generically.

There are a finite number of other such Teichmüller spaces for which we can show as above that generically $\nu(\Gamma)=0$. However in general we run into the following problem: In spite of the fact that $\nu(\Gamma)$ can be computed for all congruence groups, the difficulty in studying $\nu(\Gamma)$ on $T(\Gamma)$ is that we do not have the analogue of Theorem 6.1 for $\nu(\Gamma)$ coupled with the fact that in general $m(0, \Gamma)-\nu(\Gamma)$ may be positive. We note that $\nu\left(\Gamma_{0}(p)\right)=0$ for all primes $p$ (here $\left.\Gamma_{0}(p)=\left[\left(\begin{array}{cc}a & b \\ c & d\end{array}\right) \in \Gamma(1) ; p \mid c\right]\right)$ and that $\Gamma_{0}(p) \backslash H$ has two cusps; whereas $\nu(\Gamma(N))>0$ for $N$ large. In any event we conjecture that $m(0, \Gamma)$, and hence also $\nu(\Gamma)$, is generically zero on any nontrivial Teichmüller space and hence that generically $\Phi\left(\frac{1}{2}, \Gamma\right)$ has all its eigenvalues equal to -1 .

In the above examples we have shown that $V_{1}$ is a real analytic proper subvariety (and hence of the first Baire category). However, we still know very little about $V_{1}, V_{2}, \ldots$. We now examine a family of manifolds for which a more complete analysis is possible. Instead of deforming $\Gamma$ we deform by a character $\chi$ of $\Gamma$. The associated spectral problems are of independent interest and were examined in [PS2].

Let $\kappa_{1}, \kappa_{2}, \ldots, \kappa_{n}$ denote the inequivalent cusps of $\Gamma \backslash H$, and let $C_{1}$, $C_{2}, \ldots, C_{n}$ be the generators of the parabolic stabilizers of $\kappa_{1}, \ldots, \kappa_{n}$ respectively. For a unitary character $\chi$ of $\Gamma$ (one could just as well deal with finite-dimensional unitary representations of $\Gamma$ ) we call $\kappa_{j}$ an authentic cusp if $\chi\left(C_{j}\right)=1$, otherwise $\kappa_{j}$ is nonauthentic. We number the cusps so that $\kappa_{1}, \ldots, \kappa_{n_{1}}$ are the authentic cusps, leaving $n_{2}=n-n_{1}$ nonauthentic cusps. There is an Eisenstein series $e_{j}(z, s, \chi)$ associated with each authentic cusp. They determine the continuous and residue spectrum for the spectral problem

$$
\begin{gathered}
u(\tau z)=\chi(\tau) u(z) \text { for } z \in H \text { and } \tau \in \Gamma, \\
\Delta u+\lambda u=0 ;
\end{gathered}
$$

see for example [LP1]. The notion of a cusp form depends only on the behavior of an eigenfunction in the authentic cusps. The scattering matrix $\Phi(s, \chi)$ is defined in the usual way and is now $n_{1} \times n_{1}$. The singular set $\sigma(\Gamma, \chi)$ for (6.11) is again defined as in $\S 1$. 
The development in $\S \S 2-4$ is adapted easily to the twisted problem. For a fixed $\chi$, the energy form is given by (2.6) and (2.7) and $G$ by (2.8). The Hilbert space $H_{G}$ is the completion of $C_{0}^{(\infty)}$ automorphic data. Incoming and outgoing subspaces are defined as before, but with their support only in the authentic cusps. The wave operator, $Z(\chi)$ and its generator $B(\chi)$ are constructed from these with the obvious modifications. The previous theory can be adapted in a straightforward way to show that $B(\chi)$ has a compact resolvent, that the spectrum of $B(\chi)$ and the singular set $\sigma(\Gamma, \chi)$ coincide, and that the mini-trace formula holds [PS2].

Our main concern is with the variation of $\sigma(\Gamma, \chi)$ on deforming $\chi$. In this we shall only consider the case of most interest to us, the general case is similar. Our notation is essentially the same as that in [PS2]: $\Gamma(2)$ is freely generated by

$$
A=\left(\begin{array}{ll}
1 & 2 \\
0 & 1
\end{array}\right) \quad \text { and } \quad B=\left(\begin{array}{cc}
1 & 0 \\
-2 & 1
\end{array}\right) .
$$

For $0<\theta<1$ we set

$$
\chi_{\theta}(A)=1 \quad \text { and } \quad \chi_{\theta}(B)=\exp (2 \pi i \theta) .
$$

$F=\Gamma(2) \backslash H$ has three cusps $\{0,1, \infty\}$ but the only authentic cusp is at $\infty$. When $\theta=0$ (which is outside of our range of interest) all three cusps are authentic.

In developing a perturbation theory it is convenient to vary the operators, keeping the function space fixed. To this end we introduce the differential $\omega_{\theta}$, which has the following description in the cusps:

$$
\text { At } \infty, \omega_{\theta}=0 ; \quad \text { at } 0, \omega_{\theta}=\theta \frac{d x}{2} ; \quad \text { at } 1, \omega_{\theta}=-\theta \frac{d x}{2} .
$$

The character $\chi_{\theta}$ can now be expressed as

$$
\chi_{\theta}(\gamma)=\exp \left(2 \pi i \int_{z_{0}}^{\gamma z_{0}} \omega_{\theta}\right)
$$

We define the conjugation $U_{\theta}: L^{2}(F) \rightarrow L^{2}\left(F, \chi_{\theta}\right)$ (sending $\Gamma(2)$ automorphic data into $\omega_{0}$-twisted automorphic data) by

$$
\left(U_{\theta} f\right)(z)=\exp \left(2 \pi i \int_{z_{0}}^{z} \omega_{\theta}\right) f(z) .
$$

Under this conjugation the Laplacian becomes

$$
L(\theta) u=y^{2} \Delta_{0} u+4 \pi i\left\langle d u, \omega_{\theta}\right\rangle_{H}-4 \pi^{2}\left|\omega_{\theta}\right|_{H}^{2} u-2 \pi i \delta \omega_{\theta} u+\frac{u}{4},
$$

where

$$
\delta(p d x+q d y)=-y^{2}\left(\partial_{x} p+\partial_{y} q\right) \text { and }|p d x+q d y|_{H}^{2}=y^{2}\left(p^{2}+q^{2}\right) .
$$

The conjugate energy forms, defined by means of the partition of unity $\left\{\phi_{j}\right\}$, is equal to (2.6) in the authentic cusp at $\infty$, whereas at the cusps $\kappa_{2}$ and $\kappa_{3}$ 
at 0 and 1, respectively, they become (after transforming to the cusp to $\infty$ )

$$
\begin{aligned}
E_{j}(f)=\int \varphi_{j}\left[y\left|\partial_{y}\left(\frac{f_{1}}{y^{1 / 2}}\right)\right|^{2}+\left|\partial_{x} f_{1}\right|^{2}-(-1)^{j} 2 \pi i \theta \partial_{x} f_{1} \bar{f}_{1}\right. \\
\left.+\pi^{2} \theta^{2}\left|f_{1}\right|^{2}+\frac{\left|f_{2}\right|^{2}}{y^{2}}\right] d x d y-\int \partial_{y} \varphi_{j} \frac{\left|f_{1}\right|^{2}}{2 y} d x d y .
\end{aligned}
$$

If we expand $f_{1}$ in a Fourier series,

$$
f_{1}(z)=\sum a_{\kappa}(y) \exp (\pi i k x)
$$

the first integral on the right side of $(6.18)$ becomes

$$
\int \varphi_{j}\left[y\left|\partial_{y}\left(\frac{f_{1}}{y^{1 / 2}}\right)\right|^{2}+\frac{\left|f_{2}\right|^{2}}{y^{2}}\right] d x d y+\pi^{2} \sum_{k}\left(k+(-1)^{j} \theta\right)^{2} \int \varphi_{j}\left|a_{k}\right|^{2} d y
$$

It is now clear that the $E_{j}(\theta)$ 's are equivalent forms for $\theta$ in any compact subset of $(0,1)$. The same holds true for the $G(\theta)$ 's for a suitable choice of $c$ in $J$. As a consequence the conjugate Hilbert spaces, which are the completions of $C_{0}^{(\infty)}$ data in the $G(\theta)$ norms, are all the same; we denote this space by $H_{G}$.

The generators of the solution groups of the wave equation are of the form

$$
A_{\theta}=\left(\begin{array}{cc}
0 & I \\
L(\theta) & 0
\end{array}\right)
$$

The projections $P$ defining $Z_{\theta}$ are given by (2.19) in the authentic cusp and the generators $B_{\theta}$ are, as before, equal to $A_{\theta}$ except for the zero Fourier coefficient in the authentic cusp when $y>a$. It remains to prove that the resolvent of $B_{\theta}$ is real analytic.

Theorem 6.3. $R_{\lambda}\left(B_{\theta}\right)$ is real analytic jointly in $\lambda$ and $\theta$ for $\lambda$ in the resolvent set of $B_{\theta}$ and $\theta$ in $(0,1)$.

Proof. As shown in $\S 5$, it suffices to prove that $R_{\lambda}\left(B_{\theta}\right)$ is real analytic in $\theta$ for a single value of $\lambda>\omega^{\prime}$. Now

$$
R_{\lambda}\left(B_{\theta}\right)=R_{\lambda}\left(B_{\theta_{0}}\right)\left(I-\left(B_{\theta}-B_{\theta_{0}}\right) R_{\lambda}\left(B_{\theta_{0}}\right)\right)^{-1}
$$

when the inverse exists. Setting $\{u, v\}=R_{\lambda}\left(B_{\theta_{0}}\right) f$ we see that

$$
\lambda^{2} u-L\left(\theta_{0}\right) u=\lambda f_{1}+f_{2}
$$

and

$$
\left(B_{\theta}-B_{\theta_{0}}\right) R_{\lambda}\left(B_{\theta_{0}}\right) f=\left\{0,\left(L(\theta)-L\left(\theta_{0}\right)\right) u\right\} .
$$

Analyticity follows from elliptic estimates in any compact subset. In $\kappa_{1}$ where $L(\theta)=L\left(\theta_{0}\right)$ there is nothing to prove. In $\kappa_{j}, j=2,3$,

$$
\left(L(\theta)-L\left(\theta_{0}\right)\right) u=(-1)^{j} 2 \pi i\left(\theta-\theta_{0}\right) y^{2} \partial_{x} u-\pi^{2}\left(\theta-\theta_{0}\right)^{2} y^{2} u .
$$

It is easy to see from (6.19) that

$$
\int_{\kappa_{j}}\left|\lambda f_{1}+f_{2}\right|^{2} \frac{d x d y}{y^{2}} \leq c G(f)
$$


and by elliptic estimates as in $\S 4$ that

$$
\sum_{|\alpha| \leq 2} \int_{M}\left|\partial^{\alpha} u\right|^{2} d x d y \leq c G(f) .
$$

It now follows from Corollary 4.2 of [PS2] that

$$
\int_{\kappa_{j}} y^{4}\left(|u|^{2}+\left|\partial_{x} u\right|^{2}\right) \frac{d x d y}{y^{2}} \leq c G(f) .
$$

Combining this with (6.24) we see $\left(B_{\theta}-B_{\theta_{0}}\right) R_{\lambda}\left(B_{\theta_{0}}\right)$ is real analytic in $\theta$ and hence by (6.21) so is $R_{\lambda}\left(B_{\theta}\right)$. This completes the proof of Theorem 6.3.

Since the spectrum of $B_{\theta}$ and the singular set $i \sigma\left(\Gamma(2), \chi_{\theta}\right)$ coincide we have also established the real analyticity of the latter (except for possible algebraic singularities) for $0<\theta<1$. This fact as applied to the smallest Laplacian eigenvalue $\lambda_{0}\left(\chi_{\theta}\right)$ was needed in our paper [PS2].

We conclude this section by examining $\nu\left(\Gamma(2), \chi_{\theta}\right)$ where, in the presence of only one authentic cusp,

$$
\nu\left(\chi_{\theta}\right)=\left(\Phi\left(\frac{1}{2}, \chi_{\theta}\right)+1\right) / 2 .
$$

Thus $\nu$ is either equal to zero or one.

According to Theorem 2(ii) of [PS2] there are no cusp forms for $0<\theta<1$ with Laplacian eigenvalue in $\left[0, \frac{1}{4}\right]$, that is, with a $B_{\theta}$ eigenvalue in $\left[0, \frac{1}{2}\right]$. Hence

$$
\nu\left(\chi_{\theta}\right)=m\left(0, \Gamma(2), \chi_{\theta}\right) .
$$

As in Theorem 6.1 we have the decomposition of $(0,1)$ into real analytic sets

$$
V_{0}=(0,1), \quad V_{1}=\left\{\theta ; \nu\left(\chi_{\theta}\right) \geq 1\right\}, \quad V_{2}=\left\{\theta ; \nu\left(\chi_{\theta}\right) \geq 2\right\}=\varnothing ;
$$

the third assertion follows from Theorem 2(i) [PS2].

It remains to characterize $V_{1}$. Now $\operatorname{ker} \chi_{1 / 2} \supset \Gamma(4)$ with finite index and we showed in Case (iii) of this section that $m(0, \Gamma(4))=0$. It follows that

$$
m\left(0, \chi_{1 / 2}\right)=\nu\left(\chi_{1 / 2}\right)=0 \text {. }
$$

Thus $V_{1}$ is a proper subset of $(0,1)$, and since it is real analytic, it consists of at most a discrete set of points in $(0,1)$. In fact, $V_{1}$ is nonempty. For the eigenvalue $\lambda_{0}(\theta)$ of the Laplacian is analytic in $(0,1)$ and converges to 0 as $\theta \rightarrow 0$ (see [PS2]). Moreover $\sigma\left(\Gamma(2), \chi_{1 / 2}\right) \cap\left[0, \frac{1}{2}\right]=\varnothing$, so that by the continuity of the singular set, there is a $\theta_{0}$ in $\left(0, \frac{1}{2}\right)$ such that $\lambda_{0}\left(\theta_{0}\right)=\frac{1}{4}$ and hence $m\left(0, \chi_{\theta_{0}}\right) \geq 1$. We conclude from this that

$$
\nu\left(\chi_{\theta_{0}}\right)=1 .
$$

Presumably $V_{1}=\left\{\theta_{0}, 1-\theta_{0}\right\}$ and $\Phi\left(\frac{1}{2}, \chi_{\theta}\right)=-1$ except for $\theta=\theta_{0}$ and $1-\theta_{0}$. Actually by the numerical analysis in [PS2] we known that $\theta_{0}=\frac{1}{8} \in V_{1}$. However this does not follow from our analysis. 


\section{REFERENCES}

[B] P. Buser, Riemannsche Flachen mit eigenwerten in $(0,1 / 4)$, Comm. Math. Helv. 52 (1977), 25-34.

[E] I. Efrat, Eisenstein series and Cartan groups, Illinois J. Math. 31 (1987), 428-437.

[GK] I. Gohberg and M. Krein, Introduction to the theory of linear non-selfadjoint operators, Trans. Math. Monographs, vol. 18, Amer. Math. Soc., Providence, RI, 1969.

[He] D. Hejhal, The Selberg trace formula for $\operatorname{PSL}(2, \mathbf{R})$, vol. 2, Lecture Notes in Math., vol. 1001, Springer-Verlag, Berlin and New York, 1983.

[Hu1] M. Huxley, Introduction to Kloostermania, Banach Center Publ., vol. 17, 1985.

[Hu2] _ Scattering matrices for congruent subgroups, Modular Forms, (R. Rankin, ed.), Ellis Horwood, Chichester, 1984.

[LP1] P. Lax and R. Phillips, Scattering theory for automorphic functions, Ann. of Math. Stud., vol. 87, Princeton Univ. Press, Princeton, NJ, 1976.

[LP2] _ The time delay operator and a related trace formula, Topics in Functional Analysis (I. Gohberg and M. Kac, eds.), Academic Press, San Diego, CA, 1978.

[LP3] _ Scattering theory for automorphic functions, Bull. Amer. Math. Soc. 2 (1980), 261295.

[M] H. Maass, Uber eine neue Art von nichtanalytischen automorphismen Funktionen und die Bestimmung Dirichletscher Reihen durch Funktionalgleichungen, Math. Ann. 121 (1949), 141-183.

[PS1] R. Phillips and P. Sarnak, On cusp forms for cofinite subgroups of $\operatorname{PSL}(2, R)$, Invent. Math. 80 (1985), 339-364.

[PS2] — The spectrum of Fermat curves, GAFA 1 (1991), 80-146.

[Ra] R. Rankin, Modular forms and functions, Cambridge Univ. Press, London and New York, 1977.

[RS] M. Reed and B. Simon, Methods of mathematical physics, Vol. 4, Academic Press, San Diego, CA, 1978.

[Se] A. Selberg, Harmonic analysis, Collected works, vol. 1, Springer-Verlag, Berlin and New York, 1989.

[V] A. Venkov, Spectral theory of automorphic functions, Trudy Mat. Inst. Steklov 153 (1981), 172 pp.; English transl. in Proc. Steklov Inst. Math. (1982) no. 4, 163 pp.

[W] S. Wolpert, The spectrum of a Riemann surface with a cusp, Taniguchi Symposium Lecture, 1989.

\footnotetext{
Abstract. Let $\Gamma \subset \operatorname{PSL}(2, \mathbb{R})$ be a discrete subgroup with quotient $\Gamma \backslash H$ of finite volume but not compact. The spectrum of the Laplacian on $L^{2}$ automorphic functions is unstable under perturbations; however, it becomes much more manageable when the scattering frequencies are adjoined (with multiplicity equal to the order of the pole of the determinant of the scattering matrix at these points). This augmented set shows up in a natural way in a one-sided version of the Selberg trace formula and is the actual spectrum of the generator of a cut-off wave equation. Applying standard perturbation theory to this operator, it is proved that the augmented spectrum is real analytic in Teichmüller space. The same operator is used to derive Fermi's Golden Rule in this setting. It turns out that the proper multiplicity to be attached to the Laplacian eigenvalue at $\frac{1}{4}$ is twice the dimension of cusp forms plus $\mu=\operatorname{tr}[\Phi+I] / 2$; here $\Phi$ denotes the scattering matrix at this point. It is shown that the generic value of $\mu$ in the Teichmüller space of the once punctured torus and the six-times punctured sphere is zero. This is also true of the $\chi$-twisted spectral problem, where $\chi$ is a character for $\Gamma$.
}

Department of Mathematics, Stanford University, Stanford, California 94305

Department of Mathematics, Princeton University, Princeton, New Jersey 08544 